DOMINIK SzYNAL (Lublin)

Waldemar WoeyŃSki (Poznań)

\title{
ON SIMULATION POWERS OF NEW NORMALITY TESTS
}

Abstract. Empirical study of powers in \% of the Shapiro-Wilk test W and the new tests $T$ based on the characterization of probability distributions is presented using in simulations 100000 replications and sample sizes $n=$ $10,15,20,30,50,70$. Our tests compare favorably with the Shapiro-Wilk test and other recommended omnibus tests. The presented test statistics have a simple form and are free of restrictions required by many classical and recommended tests.

1. Introduction. Pearson et al. $[\mathrm{PE}]$ studied powers of four omnibus tests: $K^{2}$, the Bowman \& Shenton statistic; the $R$ test with rectangular contours; the Shapiro-Wilk $W$ test; and the D'Agostino $Y$ test, at the $5 \%$ level for 200 samples of sizes $n=20,50,100$ drawn from among 50 nonnormal populations specified below. The results of their paper are very useful and often applied in comparisons of the behaviour of new normality tests. Selected alternatives of Pearson et al. [PE] nonnormal populations were used in the empirical description of performance of tests in Kallenberg and Ledwina [KL, Cabaña and Cabaña [CC], Szynal and Wołyński [SW2] and in other papers. We should note that Pearson et al. [PE] used in simulation 200 samples of sizes $n=20,50,100$. Kallenberg and Ledwina [KL], Cabaña and Cabaña [CC] based on 10000 replications, while Szynal and Wołyński [SW2 applied 100000 replications in simulations of critical values and powers of tests.

In this paper we use simulations based on 100000 replications to give powers of the test $W$ under all 50 alternatives proposed by Pearson et al. [PE]. We call them $P A B$ alternatives. We compare the resulting powers with

2010 Mathematics Subject Classification: Primary 62G10; Secondary 62G30.

Key words and phrases: departure from normality, omnibus test, goodness-of-fit, record value, comparison of power.

Received 10 February 2016; revised 22 August 2016.

Published online 23 January 2017. 
the powers of the tests $T$ introduced by Szynal [S]. We also discuss Kallenberg and Ledwina's $\mathrm{KL}$ results under 10 symmetric alternatives selected from 27 PAB symmetric alternatives, which we call $K L$ symmetric alternatives, and 14 skew alternatives from the 23 skew alternatives of Pearson et al. $\mathrm{PE}$, which we call KL skew alternatives. Cabaña and Cabaña [CC] selected the same KL symmetric alternatives and 13 skew alternatives from the PAB skew alternatives, which we call $C C$ skew alternatives.

For completeness we also add the sets of 10 symmetric and 10 skew alternatives chosen by Morris and Szynal [MS2] and also discussed in Szynal and Wołyński [SW1. We call them $M S$ alternatives.

We study the alternatives taken from Pearson et al. $\mathrm{PE}$ ] PAB alternatives):

Symmetric alternatives:

1. $S B(0,0.5)[\mathrm{KL}, \mathrm{CC}, \mathrm{MS}]$,

2. Tukey (1.5) [KL, CC],

3. $\operatorname{Beta}(1,1)$,

4. $S B(0,0.707)[\mathrm{MS}]$,

5. Tukey (0.7) [KL, CC],

6. Tukey (3.0),

7. $\operatorname{Beta}(2,2)[\mathrm{MS}]$,

13. $S U(0,3)$,

14. $t_{10}$,

15. Logistic [KL, CC, MS],

16. $S U(0,2)$,

17. Tukey (10) [KL, CC],

18. Laplace [MS],

19. $S C(0.2,3)$,

20. $S C(0.05,3)[\mathrm{KL}, \mathrm{CC}$,

21. $S C(0.1,3)[\mathrm{MS}]$,

22. $S C(0.2,5)[\mathrm{KL}, \mathrm{CC}]$,

23. $S C(0.2,7)$,

24. $S C(0.1,5)[\mathrm{MS}]$,

25. $S C(0.05,5)[\mathrm{KL}, \mathrm{CC}]$,

26. $S C(0.1,7)$,

27. $S C(0.05,7)[\mathrm{KL}, \mathrm{CC}, \mathrm{MS}$,

28. $S U(0,1)[\mathrm{KL}, \mathrm{CC}]$,

29. $S U(0,0.9)$,

30. $t_{4}[\mathrm{MS}]$,

31. $t_{2}$,

32. $t_{1}[\mathrm{MS}]$,
Skew alternatives:

36. $S B(0.533,0.5)[\mathrm{KL}, \mathrm{MS}]$,

37. $\operatorname{Beta}(3,2)$,

38. $\operatorname{Beta}(2,1)[\mathrm{MS}]$,

39. $S B(1,2)$,

40. $S B(1,1)[\mathrm{KL}, \mathrm{CC}, \mathrm{MS}$,

41. $L C(0.2,3)[\mathrm{KL}, \mathrm{CC}]$,

42. $L C(0.2,5)[\mathrm{MS}]$,

43. $L C(0.2,7)$,

44. Weibull(2) [KL, CC, MS],

45. $L C(0.1,3)[\mathrm{KL}, \mathrm{CC}]$,

46. $\chi_{10}^{2}[\mathrm{KL}, \mathrm{CC}]$,

47. $L C(0.05,3)[\mathrm{KL}, \mathrm{CC}]$,

48. $L C(0.1,5)[\mathrm{KL}, \mathrm{CC}]$,

49. $S U(-1,2)[\mathrm{KL}, \mathrm{CC}]$,

50. $\chi_{4}^{2}[\mathrm{KL}, \mathrm{CC}, \mathrm{MS}]$,

51. $L C(0.1,7)$,

52. $L C(0.05,5)[\mathrm{KL}, \mathrm{CC}, \mathrm{MS}]$,

53. $\chi_{2}^{2}$,

54. $L C(0.05,7)[\mathrm{KL}, \mathrm{CC}, \mathrm{MS}]$,

55. $\chi_{1}^{2}$,

56. Weibull(0.5) [MS],

57. $S U(1,1)[\mathrm{KL}, \mathrm{CC}]$,

58. $\operatorname{LN}(0,1)[\mathrm{KL}, \mathrm{CC}, \mathrm{MS}]$, 
where

- $S B(\gamma, \delta)$ - Johnson's $S_{B}$ distribution: the law of $\frac{\exp \left(\frac{X-\gamma}{\delta}\right)}{1+\exp \left(\frac{X-\gamma}{\delta}\right)}, X \sim N(0,1)$,

- Tukey $(\lambda)$ - Tukey's distribution: the law of $U^{\lambda}-(1-U)^{\lambda}, U$ uniform on $[0,1]$,

- $\operatorname{Beta}(a, b)$ - beta distribution:

$$
f(x)=[\operatorname{Beta}(a, b)]^{-1} x^{a-1}(1-x)^{b-1}, \quad 0 \leq x \leq 1,
$$

- $S U(\gamma, \delta)$ - Johnson's $S_{U}$ distribution: the law of $\sinh \left(\frac{X-\gamma}{\delta}\right), X \sim N(0,1)$,

- $t_{n}$ - Student's $t$ distribution with $n$ degrees of freedom:

$f(x)=\Gamma\left[\frac{1}{2}(n+1)\right]\left[\Gamma\left(\frac{1}{2} n\right)\right]^{-1}(n \pi)^{-1 / 2}\left(1+x^{2} / n\right)^{-(n+1) / 2}, \quad-\infty<x<\infty$,

- Logistic - logistic distribution: the law of $\log \frac{U}{1-U}, U$ uniform on $[0,1]$,

- Laplace - Laplace distribution:

$$
f(x)=\frac{1}{2} \exp (-|x|), \quad-\infty<x<\infty,
$$

- $S C(p, \lambda)$ - scale contaminated distribution:

$f(x)=(2 \pi)^{-1 / 2}\left[(1-p) \exp \left(-\frac{x^{2}}{2}\right)+\frac{p}{\lambda} \exp \left(-\frac{x^{2} \lambda^{-2}}{2}\right)\right], \quad-\infty<x<\infty$,

- $L C(p, \mu)$ - location contaminated distribution:

$f(x)=(2 \pi)^{-1 / 2}\left[(1-p) \exp \left(-\frac{x^{2}}{2}\right)+p \exp \left(-\frac{(x-\mu)^{2}}{2}\right)\right], \quad-\infty<x<\infty$,

- Weibull $(\theta)$ - Weibull distribution:

$$
f(x)=\theta x^{\theta-1} \exp \left(-x^{\theta}\right), \quad 0<x<\infty,
$$

- $\chi_{n}^{2}$ - chi-square distribution with $n$ degrees of freedom:

$$
f(x)=\frac{1}{2^{n / 2} \Gamma(n / 2)} x^{n / 2-1} \exp (-x / 2), \quad 0<x<\infty,
$$

- $L N(\gamma, \delta)$ - lognormal distribution: the law of $\exp \left(\frac{X-\gamma}{\delta}\right), X \sim N(0,1)$.

We need here the results of simulations of Pearson et al. [PE] under the $\mathrm{PAB}, \mathrm{KL}, \mathrm{CC}$ and MS alternatives. They are given in Section 3 .

2. The family of tests for normality. Goodness-of-fit tests derived from characterizations of continuous distributions via record values were given in Morris and Szynal [MS2]. The simulation powers of those tests were 
discussed by Szynal and Wołyński [SW1], [SW2]. Some modification of those tests was proposed by Szynal [S]. To verify $H_{0}: X \sim N(\mu, \sigma)$ using the latter tests we need the following quantities and test statistics:

$$
\begin{aligned}
a_{n}^{(r, k)}= & \frac{1}{n}\left[\frac{\Gamma(2 r+5)}{(2 k-1)^{2 r+5}}-\frac{\Gamma^{2}(r+3)}{k^{2 r+6}}\right], \\
b_{n}^{(r, k)}= & \frac{k}{n}\left[\frac{\Gamma(2 r+5)}{(k-1)^{r+1} k^{r+3}} B_{\frac{k-1}{2 k-1}}(r+2, r+3)\right. \\
& \left.+\frac{\Gamma(2 r+4)}{(2 k-1)^{2 r+4}}-\frac{\Gamma(r+2) \Gamma(r+3)}{k^{2 r+4}}\right], \\
c_{n}^{(r, k)}= & \frac{1}{\left(\begin{array}{l}
n \\
k
\end{array}\right)} \sum_{j=1}^{k-1}\left(\begin{array}{l}
k \\
j
\end{array}\right)\left(\begin{array}{l}
n-k \\
k-j
\end{array}\right)\left[2 \frac{\Gamma(2 r+4)}{k^{r+1}(k-j)^{r+1}} B_{\frac{k-j}{2 k-j}}(r+2, r+2)\right. \\
& \left.+j \frac{\Gamma(2 r+3)}{(2 k-j)^{2 r+3}}-\frac{\Gamma^{2}(r+2)}{k^{2 r+2}}\right]+\frac{1}{\left(\begin{array}{l}
n \\
k
\end{array}\right)}\left[\frac{\Gamma(2 r+3)-\Gamma^{2}(r+2)}{k^{2 r+2}}\right],
\end{aligned}
$$

where $k \in \mathbb{N}, r \neq 0$, and

$$
B_{x}(\alpha, \beta)=\int_{0}^{x} t^{\alpha-1}(1-t)^{\beta-1} d t, \quad 0<x<1, \alpha, \beta>0,
$$

denotes the incomplete beta function;

$$
\begin{aligned}
& E_{1}^{(r, k)}=\mathrm{E}\left[\phi(Z)(1-\Phi(Z))^{k-2} \log ^{r} \frac{1}{1-\Phi(Z)}\right], \quad Z \sim N(0,1), \\
& E_{2}^{(r, k)}=\mathrm{E}\left[Z \phi(Z)(1-\Phi(Z))^{k-2} \log ^{r} \frac{1}{1-\Phi(Z)}\right],
\end{aligned}
$$

where $\phi(x)=(2 \pi)^{-1 / 2} e^{-x^{2} / 2}, \Phi(x)=\int_{-\infty}^{x} \phi(t) d t$;

$$
\begin{aligned}
s_{n}^{(r, k)}= & \frac{1}{n}\left[\left((k-1) E_{1}^{(r+2, k)}-(r+2) E_{1}^{(r+1, k)}\right)^{2}\right. \\
& \left.+\frac{1}{2}\left((k-1) E_{2}^{(r+2, k)}-(r+2) E_{2}^{(r+1, k)}\right)^{2}\right], \\
t_{n}^{(r, k)}= & \frac{k(r+1)}{n}\left[(r+2)\left(E_{1}^{(r, k)} E_{1}^{(r+1, k)}+\frac{1}{2} E_{2}^{(r, k)} E_{2}^{(r+1, k)}\right)\right. \\
& \left.-(k-1)\left(E_{1}^{(r, k)} E_{1}^{(r+2, k)}+\frac{1}{2} E_{2}^{(r, k)} E_{2}^{(r+2, k)}\right)\right], \\
u_{n}^{(r, k)}= & \frac{k^{2}(r+1)^{2}}{n}\left[\left(E_{1}^{(r, k)}\right)^{2}+\frac{1}{2}\left(E_{2}^{(r, k)}\right)^{2}\right], \\
a_{n 1}^{(r, k)}= & a_{n}^{(r, k)}-s_{n}^{(r, k)},
\end{aligned}
$$




$$
\begin{aligned}
& b_{n 1}^{(r, k)}=b_{n}^{(r, k)}-t_{n}^{(r, k)}, \\
& c_{n 1}^{(r, k)}=c_{n}^{(r, k)}-u_{n}^{(r, k)}, \\
& \Delta_{n 1}^{(r, k)}=\operatorname{det}\left[\begin{array}{cc}
a_{n 1}^{(r, k)} & b_{n 1}^{(r, k)} \\
b_{n 1}^{(r, k)} & c_{n 1}^{(r, k)}
\end{array}\right] \\
& \hat{T}_{n}^{(r, k)}=\frac{1}{\Delta_{n 1}^{(r, k)}}\left[c _ { n 1 } ^ { ( r , k ) } \left(\frac{1}{n} \sum_{i=1}^{n}\left(1-\Phi\left(\frac{X_{i: n}-\bar{X}_{n}}{S_{n}}\right)\right)^{k-1}\right.\right. \\
& \left.\times \log \left(1-\Phi\left(\frac{X_{i: n}-\bar{X}_{n}}{S_{n}}\right)\right)^{r+2}-\frac{\Gamma(r+3)}{k^{r+3}}\right)^{2} \\
& -2 b_{n 1}^{(r, k)}\left(\frac{1}{n} \sum_{i=1}^{n}\left(1-\Phi\left(\frac{X_{i: n}-\bar{X}_{n}}{S_{n}}\right)\right)^{k-1}\right. \\
& \left.\times \log \left(1-\Phi\left(\frac{X_{i: n}-\bar{X}_{n}}{S_{n}}\right)\right)^{r+2}-\frac{\Gamma(r+3)}{k^{r+3}}\right) \\
& \times\left(\frac{1}{\left(\begin{array}{l}
n \\
k
\end{array}\right)} \sum_{i=1}^{n-k+1}\left(\begin{array}{l}
n-i \\
k-1
\end{array}\right) \log \left(1-\Phi\left(\frac{X_{i: n}-\bar{X}_{n}}{S_{n}}\right)\right)^{r+1}-\frac{\Gamma(r+2)}{k^{r+1}}\right) \\
& \left.+a_{n 1}^{(r, k)}\left(\frac{1}{\left(\begin{array}{l}
n \\
k
\end{array}\right)} \sum_{i=1}^{n-k+1}\left(\begin{array}{l}
n-i \\
k-1
\end{array}\right) \log \left(1-\Phi\left(\frac{X_{i: n}-\bar{X}_{n}}{S_{n}}\right)\right)^{r+1}-\frac{\Gamma(r+2)}{k^{r+1}}\right)^{2}\right] \\
& =\hat{T}_{n ; c_{1}}^{(r, k)}+\hat{T}_{n ; c_{2}}^{(r, k)}=\hat{T}_{n ; c_{3}}^{(r, k)}+\hat{T}_{n ; c_{4}}^{(r, k)},
\end{aligned}
$$

where $\bar{X}_{n}=n^{-1} \sum_{i=1}^{n} X_{i}, S_{n}^{2}=n^{-1} \sum_{i=1}^{n}\left(X_{i}-\bar{X}_{n}\right)^{2}$,

$$
\begin{aligned}
& \hat{T}_{n ; c_{1}}^{(r, k)}=\frac{1}{a_{n 1}^{(r, k)}}\left[\frac{1}{n} \sum_{i=1}^{n}\left(1-\Phi\left(\frac{X_{i: n}-\bar{X}_{n}}{S_{n}}\right)\right)^{k-1}\right. \\
& \left.\times \log \left(1-\Phi\left(\frac{X_{i: n}-\bar{X}_{n}}{S_{n}}\right)\right)^{r+2}-\frac{\Gamma(r+3)}{k^{r+3}}\right]^{2}, \\
& \hat{T}_{n ; c_{2}}^{(r, k)}=\frac{1}{\Delta_{n 1}^{(r, k)} a_{n 1}^{(r, k)}}\left[a_{n 1}^{(r, k)} \frac{1}{\left(\begin{array}{l}
n \\
k
\end{array}\right)} \sum_{i=1}^{n-k+1}\left(\begin{array}{c}
n-i \\
k-1
\end{array}\right) \log \left(1-\Phi\left(\frac{X_{i: n}-\bar{X}_{n}}{S_{n}}\right)\right)^{r+1}\right. \\
& -b_{n 1}^{(r, k)} \frac{1}{n} \sum_{i=1}^{n}\left(1-\Phi\left(\frac{X_{i: n}-\bar{X}_{n}}{S_{n}}\right)\right)^{k-1} \log \left(1-\Phi\left(\frac{X_{i: n}-\bar{X}_{n}}{S_{n}}\right)\right)^{r+2} \\
& \left.-\frac{\Gamma(r+2)}{k^{r+3}}\left(k^{2} a_{n 1}^{(r, k)}-(r+2) b_{n 1}^{(r, k)}\right)\right]^{2} \\
& \hat{T}_{n ; c_{3}}^{(r, k)}=\frac{1}{c_{n 1}^{(r, k)}}\left[\frac{1}{\left(\begin{array}{l}
n \\
k
\end{array}\right)} \sum_{i=1}^{n-k+1}\left(\begin{array}{c}
n-i \\
k-1
\end{array}\right) \log \left(1-\Phi\left(\frac{X_{i: n}-\bar{X}_{n}}{S_{n}}\right)\right)^{r+1}-\frac{\Gamma(r+2)}{k^{r+1}}\right]^{2},
\end{aligned}
$$




$$
\begin{aligned}
\hat{T}_{n ; c_{4}}^{(r, k)}= & \frac{1}{\Delta_{n 1}^{(r, k)} c_{n 1}^{(r, k)}} \\
\times & {\left[c_{n 1}^{(r, k)} \frac{1}{n} \sum_{i=1}^{n}\left(1-\Phi\left(\frac{X_{i: n}-\bar{X}_{n}}{S_{n}}\right)\right)^{k-1} \log \left(1-\Phi\left(\frac{X_{i: n}-\bar{X}_{n}}{S_{n}}\right)\right)^{r+2}\right.} \\
& -b_{n 1}^{(r, k)} \frac{1}{\left(\begin{array}{l}
n \\
k
\end{array}\right)} \sum_{i=1}^{n-k+1}\left(\begin{array}{l}
n-i \\
k-1
\end{array}\right) \log \left(1-\Phi\left(\frac{X_{i: n}-\bar{X}_{n}}{S_{n}}\right)\right)^{r+1} \\
& \left.-\frac{\Gamma(r+2)}{k^{r+3}}\left((r+2) c_{n 1}^{(r, k)}-k^{2} b_{n 1}^{(r, k)}\right)\right]^{2} .
\end{aligned}
$$

3. Simulation results for powers of the five presented tests. For an empirical comparison of performance of the tests proposed by Szynal [S] with the recommended ones and also with the test $W$ in Pearson et al. $[\mathrm{PE}]$ we have taken their alternatives. Moreover, we do comparisons of powers using selected KL, CC and MS alternatives. Samples of size $n=10,15,20,30,50,70$ are taken with $k=1,2,3,4,5$ and $r=$ $-1.49,-1.47,-1.45,-1.43,-1.4,-1.35,-1.3,-1.25,-1.2,-1.1,-1.01,-0.99$, $-0.95,-0.9,-0.8,-0.7,-0.6,-0.5,-0.4,-0.3,-0.1,0.1,0.3,0.5,0.7,0.9,1.0$, $1.2,1.25,1.3,1.35,1.4,1.45,1.5,1.6,1.7,2.0$.

Critical values were simulated using 100000 replications and associated powers were obtained using 100000 replications but only some results are presented here (all simulations are available from the second author).

The performance of our tests $T$ and the test $W$ under different alternatives is shown in the tables below. We give characters of $T$ with $k=$ $1,2,3,4,5$ showing only the maximum average powers.

Table 1. Symmetric PAB populations; sample size $n=10,15,20,30,50,70$; estimates of power of $W$ and $T$ based on 100000 replications

\begin{tabular}{|c|r|r|r|r|r|r|}
\hline$n$ & \multicolumn{6}{|c|}{10} \\
\hline Alt. $\backslash$ Test & \multicolumn{1}{|c|}{$W$} & $\hat{T}_{10}^{(-1.25,1)}$ & $\hat{T}_{10}^{(-1.25,2)}$ & $\hat{T}_{10}^{(-1.01,3)}$ & $\hat{T}_{10}^{(-1.01,4)}$ & $\hat{T}_{10}^{(0.5,5)}$ \\
\hline 1 & 16.05 & 10.88 & 17.38 & 11.64 & 8.01 & 20.89 \\
2 & 9.74 & 7.18 & 11.89 & 7.65 & 5.80 & 15.38 \\
3 & 8.31 & 6.24 & 10.42 & 6.87 & 5.09 & 13.72 \\
4 & 6.87 & 5.42 & 8.90 & 5.84 & 4.49 & 12.17 \\
5 & 5.78 & 4.84 & 7.95 & 5.24 & 4.09 & 10.71 \\
6 & 4.41 & 3.53 & 5.71 & 3.85 & 3.40 & 7.84 \\
7 & 4.23 & 3.52 & 5.32 & 3.85 & 3.51 & 7.52 \\
13 & 6.30 & 6.76 & 6.30 & 6.70 & 6.44 & 5.24 \\
14 & 7.21 & 7.95 & 7.42 & 7.79 & 7.58 & 5.42 \\
15 & 8.11 & 8.92 & 8.12 & 8.89 & 8.53 & 5.81 \\
16 & 8.31 & 9.29 & 8.61 & 9.11 & 8.78 & 5.97 \\
17 & 52.33 & 38.34 & 38.36 & 44.07 & 39.48 & 35.74 \\
\hline
\end{tabular}


Table 1 (cont.)

\begin{tabular}{|c|c|c|c|c|c|c|}
\hline$n$ & \multicolumn{6}{|c|}{10} \\
\hline Alt. \Test & $W$ & $\hat{T}_{10}^{(-1.25,1)}$ & $\hat{T}_{10}^{(-1.25,2)}$ & $\hat{T}_{10}^{(-1.01,3)}$ & $\hat{T}_{10}^{(-1.01,4)}$ & $\hat{T}_{10}^{(0.5,5)}$ \\
\hline 18 & 15.32 & 16.65 & 15.00 & 16.71 & 15.56 & 9.83 \\
\hline 19 & 19.59 & 21.27 & 19.90 & 21.49 & 19.89 & 12.19 \\
\hline 20 & 11.23 & 12.27 & 11.44 & 12.12 & 11.34 & 7.95 \\
\hline 21 & 15.04 & 17.05 & 15.69 & 16.87 & 15.48 & 10.10 \\
\hline 22 & 40.08 & 39.67 & 37.75 & 40.95 & 37.24 & 26.55 \\
\hline 23 & 52.83 & 50.53 & 48.33 & 52.76 & 47.89 & 38.02 \\
\hline 24 & 30.01 & 31.18 & 29.95 & 31.45 & 28.97 & 21.33 \\
\hline 25 & 19.66 & 20.80 & 20.23 & 20.52 & 19.67 & 14.72 \\
\hline 26 & 39.97 & 39.78 & 38.39 & 40.72 & 37.63 & 29.85 \\
\hline 27 & 25.55 & 26.32 & 25.71 & 26.37 & 24.92 & 20.16 \\
\hline 28 & 23.40 & 24.97 & 23.11 & 24.91 & 23.41 & 15.22 \\
\hline 29 & 27.74 & 29.15 & 27.49 & 29.49 & 27.43 & 18.86 \\
\hline 30 & 13.69 & 15.38 & 13.97 & 15.00 & 14.41 & 9.10 \\
\hline 31 & 29.68 & 31.27 & 29.41 & 31.33 & 29.26 & 20.65 \\
\hline 32 & 59.19 & 57.86 & 56.06 & 59.22 & 55.34 & 47.18 \\
\hline Av. & 20.76 & 20.26 & 20.33 & 20.79 & 19.02 & 16.60 \\
\hline$n$ & \multicolumn{6}{|c|}{15} \\
\hline Alt. \Test & $W$ & $\hat{T}_{15 ; c_{4}}^{(-0.6,1)}$ & $\hat{T}_{15}^{(-1.3,2)}$ & $\hat{T}_{15}^{(-0.95,3)}$ & $\hat{T}_{15}^{(-0.1,4)}$ & $\hat{T}_{15}^{(0.5,5)}$ \\
\hline 1 & 28.48 & 32.34 & 27.56 & 22.24 & 34.31 & 32.63 \\
\hline 2 & 15.98 & 21.61 & 17.36 & 13.26 & 24.21 & 22.96 \\
\hline 3 & 12.67 & 18.50 & 14.59 & 11.35 & 20.90 & 20.16 \\
\hline 4 & 9.73 & 15.54 & 12.14 & 9.29 & 18.27 & 17.47 \\
\hline 5 & 7.79 & 12.74 & 9.89 & 7.56 & 15.57 & 15.07 \\
\hline 6 & 4.97 & 7.80 & 5.87 & 4.60 & 9.63 & 9.62 \\
\hline 7 & 4.52 & 6.83 & 5.34 & 4.47 & 9.18 & 9.22 \\
\hline 13 & 6.89 & 7.02 & 7.46 & 7.38 & 5.82 & 5.78 \\
\hline 14 & 8.54 & 8.69 & 9.47 & 9.21 & 6.88 & 6.60 \\
\hline 15 & 9.84 & 10.08 & 11.01 & 10.88 & 7.93 & 7.38 \\
\hline 16 & 10.45 & 10.71 & 11.66 & 11.30 & 8.04 & 7.70 \\
\hline 17 & 68.48 & 47.95 & 55.06 & 56.29 & 60.05 & 62.74 \\
\hline 18 & 20.91 & 20.84 & 23.45 & 23.49 & 17.72 & 17.34 \\
\hline 19 & 29.07 & 29.61 & 31.87 & 31.67 & 23.39 & 21.97 \\
\hline 20 & 15.39 & 15.80 & 16.68 & 16.24 & 12.26 & 11.75 \\
\hline 21 & 22.51 & 23.34 & 24.61 & 24.00 & 17.61 & 16.78 \\
\hline 22 & 57.72 & 55.35 & 59.24 & 59.94 & 51.69 & 50.23 \\
\hline 23 & 72.99 & 68.44 & 72.64 & 73.08 & 67.64 & 66.77 \\
\hline 24 & 43.61 & 44.02 & 46.04 & 45.36 & 38.37 & 37.05 \\
\hline 25 & 28.40 & 29.05 & 30.21 & 29.51 & 24.81 & 23.84 \\
\hline 26 & 55.68 & 55.19 & 57.24 & 56.73 & 51.37 & 50.19 \\
\hline 27 & 36.16 & 36.88 & 37.70 & 36.88 & 33.45 & 32.33 \\
\hline 28 & 33.45 & 33.58 & 36.46 & 36.25 & 29.22 & 27.93 \\
\hline 29 & 40.04 & 39.80 & 42.56 & 43.24 & 35.72 & 34.40 \\
\hline 30 & 19.08 & 19.57 & 21.67 & 21.08 & 15.59 & 14.94 \\
\hline 31 & 42.66 & 42.34 & 45.42 & 45.31 & 37.85 & 36.94 \\
\hline 32 & 76.30 & 74.16 & 77.16 & 76.91 & 73.61 & 73.18 \\
\hline Av. & 28.97 & 29.18 & 30.01 & 29.17 & 27.82 & 27.15 \\
\hline
\end{tabular}


Table 1 (cont.)

\begin{tabular}{|c|c|c|c|c|c|c|}
\hline$n$ & \multicolumn{6}{|c|}{20} \\
\hline Alt. \Test & $W$ & $\begin{array}{l}\hat{T}_{20 ; c_{4}}^{(-0.6,1)} \\
\end{array}$ & $\hat{T}_{20}^{(-1.25,2)}$ & $\hat{T}_{20}^{(-0.6,3)}$ & $\hat{T}_{20}^{(0.1,4)}$ & $\hat{T}_{20}^{(0.7,5)}$ \\
\hline 1 & 44.10 & 46.75 & 38.34 & 51.95 & 48.92 & 45.70 \\
\hline 2 & 24.95 & 31.28 & 23.88 & 36.47 & 34.16 & 31.49 \\
\hline 3 & 19.72 & 26.79 & 20.08 & 31.68 & 29.46 & 27.68 \\
\hline 4 & 14.74 & 21.76 & 15.96 & 26.79 & 25.19 & 23.86 \\
\hline 5 & 11.52 & 17.70 & 12.67 & 22.34 & 21.15 & 19.54 \\
\hline 6 & 6.20 & 10.14 & 6.75 & 12.94 & 12.50 & 11.42 \\
\hline 7 & 5.47 & 8.37 & 5.74 & 11.61 & 11.52 & 10.88 \\
\hline 13 & 7.67 & 8.06 & 8.72 & 6.48 & 6.80 & 6.07 \\
\hline 14 & 9.86 & 10.49 & 11.57 & 8.24 & 8.13 & 7.44 \\
\hline 15 & 11.61 & 12.40 & 13.88 & 9.80 & 9.74 & 8.88 \\
\hline 16 & 12.60 & 13.68 & 14.92 & 10.50 & 10.45 & 9.45 \\
\hline 17 & 80.59 & 59.91 & 68.42 & 73.52 & 76.67 & 79.62 \\
\hline 18 & 25.92 & 28.32 & 31.42 & 25.33 & 25.91 & 24.61 \\
\hline 19 & 37.38 & 39.89 & 43.52 & 33.65 & 33.38 & 30.72 \\
\hline 20 & 19.26 & 20.46 & 21.89 & 16.42 & 16.04 & 14.49 \\
\hline 21 & 28.74 & 30.50 & 32.87 & 24.69 & 24.04 & 21.56 \\
\hline 22 & 70.62 & 70.81 & 74.53 & 67.85 & 67.82 & 66.21 \\
\hline 23 & 84.83 & 82.96 & 86.04 & 82.60 & 82.46 & 81.56 \\
\hline 24 & 54.40 & 56.32 & 58.33 & 51.09 & 50.52 & 48.10 \\
\hline 25 & 36.09 & 37.11 & 38.30 & 32.87 & 32.44 & 30.45 \\
\hline 26 & 67.32 & 68.13 & 69.78 & 64.14 & 64.31 & 62.16 \\
\hline 27 & 45.16 & 46.10 & 46.78 & 42.55 & 42.46 & 40.58 \\
\hline 28 & 42.44 & 44.76 & 48.02 & 40.85 & 41.15 & 39.17 \\
\hline 29 & 50.45 & 51.95 & 55.34 & 48.98 & 49.29 & 47.37 \\
\hline 30 & 24.09 & 26.00 & 28.13 & 21.38 & 21.36 & 19.53 \\
\hline 31 & 52.89 & 55.00 & 57.98 & 51.50 & 51.21 & 49.44 \\
\hline 32 & 86.77 & 86.29 & 88.20 & 86.13 & 86.40 & 85.94 \\
\hline Av. & 36.12 & 37.48 & 37.85 & 36.75 & 36.43 & 34.96 \\
\hline$n$ & \multicolumn{6}{|c|}{30} \\
\hline Alt. $\backslash$ Test & $W$ & $\hat{T}_{30 ; c_{4}}^{(-0.6,1)}$ & $\hat{T}_{30 ; c_{1}}^{(-1.3,2)}$ & $\hat{T}_{30}^{(-0.6,3)}$ & $\hat{T}_{30}^{(0.1,4)}$ & $\hat{T}_{30}^{(0.5,5)}$ \\
\hline 1 & 73.01 & 73.02 & 75.97 & 74.69 & 69.91 & 66.34 \\
\hline 2 & 47.46 & 55.02 & 57.94 & 56.94 & 50.83 & 48.29 \\
\hline 3 & 38.20 & 47.97 & 51.44 & 50.09 & 44.49 & 42.05 \\
\hline 4 & 27.37 & 39.11 & 43.10 & 42.26 & 37.57 & 36.15 \\
\hline 5 & 21.19 & 32.15 & 35.77 & 35.77 & 31.12 & 29.49 \\
\hline 6 & 10.95 & 17.92 & 20.14 & 19.79 & 16.73 & 15.92 \\
\hline 7 & 7.85 & 13.77 & 16.62 & 17.07 & 15.27 & 15.36 \\
\hline 13 & 8.95 & 9.52 & 8.54 & 7.74 & 7.32 & 7.00 \\
\hline 14 & 11.83 & 13.22 & 11.75 & 10.33 & 9.94 & 9.29 \\
\hline 15 & 14.80 & 16.61 & 15.02 & 13.15 & 12.66 & 11.69 \\
\hline 16 & 15.66 & 17.85 & 15.85 & 14.11 & 13.61 & 12.47 \\
\hline 17 & 94.08 & 76.87 & 83.92 & 90.90 & 92.71 & 94.74 \\
\hline 18 & 35.69 & 39.63 & 39.91 & 39.38 & 39.91 & 38.37 \\
\hline 19 & 51.10 & 55.89 & 54.08 & 50.01 & 48.63 & 45.83 \\
\hline
\end{tabular}


Table 1 (cont.)

\begin{tabular}{|c|c|c|c|c|c|c|}
\hline$n$ & \multicolumn{6}{|c|}{30} \\
\hline Alt. $\backslash$ Test & $W$ & $\hat{T}_{20 ; c_{4}}^{(-0.6,1)}$ & $\hat{T}_{20}^{(-1.25,2)}$ & $\hat{T}_{20}^{(-0.6,3)}$ & $\hat{T}_{20}^{(0.1,4)}$ & $\hat{T}_{20}^{(0.7,5)}$ \\
\hline 20 & 26.66 & 27.54 & 25.93 & 22.70 & 21.24 & 19.59 \\
\hline 21 & 39.73 & 42.38 & 39.99 & 35.59 & 33.94 & 31.46 \\
\hline 22 & 86.31 & 87.70 & 87.79 & 86.08 & 85.67 & 84.94 \\
\hline 23 & 95.41 & 95.03 & 95.57 & 95.07 & 94.82 & 94.71 \\
\hline 24 & 70.20 & 71.66 & 70.55 & 67.96 & 66.36 & 64.31 \\
\hline 25 & 48.66 & 49.10 & 47.94 & 45.44 & 44.14 & 42.12 \\
\hline 26 & 81.95 & 81.45 & 81.86 & 80.13 & 79.37 & 78.21 \\
\hline 27 & 59.21 & 58.16 & 58.61 & 56.34 & 55.40 & 54.47 \\
\hline 28 & 57.01 & 60.94 & 60.58 & 59.16 & 58.84 & 57.24 \\
\hline 29 & 65.85 & 68.85 & 69.39 & 68.43 & 68.18 & 67.07 \\
\hline 30 & 32.56 & 36.13 & 34.45 & 31.64 & 30.62 & 28.75 \\
\hline 31 & 68.62 & 69.79 & 71.52 & 69.83 & 69.75 & 68.41 \\
\hline 32 & 95.81 & 84.59 & 96.39 & 96.52 & 96.63 & 96.56 \\
\hline Av. & 47.63 & 49.70 & 50.76 & 49.52 & 47.99 & 46.70 \\
\hline$n$ & \multicolumn{6}{|c|}{50} \\
\hline Alt. $\backslash$ Test & $W$ & $\hat{T}_{50 ; c_{2}}^{(-0.7,1)}$ & $\hat{T}_{50 ; c_{1}}^{(-1.3,2)}$ & $\hat{T}_{50}^{(-0.6,3)}$ & $\hat{T}_{50}^{(-0.1,4)}$ & $\hat{T}_{50}^{(0.7,5)}$ \\
\hline 1 & 97.14 & 94.80 & 96.24 & 95.09 & 91.97 & 90.34 \\
\hline 2 & 84.01 & 83.47 & 87.31 & 83.76 & 77.68 & 73.35 \\
\hline 3 & 74.86 & 77.09 & 81.62 & 77.44 & 71.26 & 66.45 \\
\hline 4 & 57.98 & 66.43 & 73.44 & 69.10 & 62.47 & 57.92 \\
\hline 5 & 47.77 & 56.85 & 64.53 & 59.95 & 52.73 & 48.85 \\
\hline 6 & 26.31 & 31.98 & 39.32 & 34.60 & 28.45 & 24.39 \\
\hline 7 & 15.11 & 22.77 & 31.52 & 29.18 & 24.98 & 23.32 \\
\hline 13 & 10.76 & 13.00 & 10.56 & 9.51 & 8.99 & 8.19 \\
\hline 14 & 15.69 & 19.55 & 16.17 & 14.43 & 13.40 & 11.85 \\
\hline 15 & 19.28 & 25.20 & 21.98 & 19.59 & 18.56 & 16.95 \\
\hline 16 & 21.39 & 27.38 & 23.44 & 21.14 & 20.07 & 18.01 \\
\hline 17 & 99.68 & 90.50 & 97.26 & 99.20 & 99.56 & 99.67 \\
\hline 18 & 52.11 & 59.51 & 60.87 & 61.53 & 61.18 & 60.91 \\
\hline 19 & 70.49 & 77.99 & 75.56 & 71.95 & 69.80 & 65.67 \\
\hline 20 & 37.77 & 42.05 & 36.77 & 33.38 & 31.20 & 26.90 \\
\hline 21 & 56.08 & 62.16 & 57.16 & 52.73 & 49.77 & 45.00 \\
\hline 22 & 97.14 & 97.94 & 97.87 & 97.53 & 97.17 & 96.35 \\
\hline 23 & 99.58 & 99.65 & 99.61 & 99.52 & 99.50 & 99.35 \\
\hline 24 & 86.95 & 89.00 & 87.24 & 85.50 & 84.07 & 81.32 \\
\hline 25 & 66.23 & 68.49 & 65.07 & 62.70 & 60.70 & 57.24 \\
\hline 26 & 94.21 & 95.04 & 94.19 & 93.42 & 92.88 & 91.56 \\
\hline 27 & 76.59 & 78.30 & 75.95 & 74.62 & 73.25 & 70.80 \\
\hline 28 & 76.54 & 81.40 & 82.31 & 80.93 & 80.11 & 78.88 \\
\hline 29 & 84.44 & 88.14 & 88.98 & 88.40 & 88.41 & 87.55 \\
\hline 30 & 46.92 & 54.67 & 51.67 & 48.59 & 46.78 & 43.72 \\
\hline 31 & 86.15 & 89.49 & 89.71 & 89.11 & 88.41 & 87.73 \\
\hline 32 & 99.64 & 99.67 & 99.77 & 99.76 & 99.79 & 99.81 \\
\hline Av. & 62.99 & 66.39 & 66.89 & 64.91 & 62.71 & 60.45 \\
\hline
\end{tabular}


Table 1 (cont.)

\begin{tabular}{|c|c|c|c|c|c|c|}
\hline$n$ & \multicolumn{6}{|c|}{70} \\
\hline Alt. $\backslash$ Test & $W$ & $\hat{T}_{70 ; c_{4}}^{(-0.99,1)}$ & $\hat{T}_{70 ; c_{1}}^{(-1.2)}$ & $\hat{T}_{70}^{(-0.6,3)}$ & $\hat{T}_{70 ; c_{3}}^{(-0.3,4)}$ & $\hat{T}_{70 ; c_{3}}^{(-0.5)}$ \\
\hline 1 & 99.89 & 94.91 & 99.55 & 99.30 & 95.94 & 92.87 \\
2 & 97.37 & 88.35 & 97.03 & 95.12 & 94.96 & 92.42 \\
3 & 93.67 & 83.87 & 94.51 & 91.70 & 93.17 & 90.61 \\
4 & 82.28 & 76.59 & 89.77 & 85.79 & 89.67 & 86.72 \\
5 & 73.22 & 68.88 & 83.36 & 77.73 & 84.91 & 82.10 \\
6 & 47.03 & 44.46 & 56.97 & 49.32 & 64.44 & 62.06 \\
7 & 25.76 & 32.18 & 46.96 & 41.55 & 53.99 & 51.60 \\
13 & 12.14 & 15.41 & 12.32 & 11.37 & 8.44 & 7.69 \\
14 & 18.61 & 23.56 & 20.21 & 17.97 & 13.36 & 12.48 \\
15 & 24.30 & 30.88 & 27.97 & 25.32 & 18.78 & 17.29 \\
16 & 26.53 & 34.01 & 30.45 & 27.42 & 20.71 & 19.63 \\
17 & 99.98 & 94.19 & 99.67 & 99.94 & 99.86 & 99.78 \\
18 & 65.24 & 70.21 & 75.04 & 76.00 & 63.11 & 61.46 \\
19 & 83.00 & 88.13 & 87.29 & 84.32 & 78.27 & 76.68 \\
20 & 47.17 & 52.38 & 45.23 & 42.16 & 37.02 & 35.17 \\
21 & 67.75 & 73.57 & 68.81 & 64.52 & 58.17 & 56.11 \\
22 & 99.45 & 99.62 & 99.61 & 99.48 & 99.16 & 99.03 \\
23 & 99.97 & 99.98 & 99.96 & 99.94 & 99.95 & 99.90 \\
24 & 94.11 & 95.56 & 94.35 & 93.29 & 91.48 & 90.97 \\
25 & 77.47 & 79.80 & 76.10 & 74.19 & 70.72 & 69.51 \\
26 & 98.15 & 98.54 & 98.07 & 97.82 & 97.25 & 97.00 \\
27 & 86.67 & 87.91 & 86.09 & 84.94 & 82.77 & 82.27 \\
28 & 87.61 & 90.48 & 91.91 & 91.47 & 86.02 & 84.94 \\
29 & 93.53 & 94.92 & 96.08 & 95.98 & 92.59 & 92.06 \\
30 & 58.10 & 65.52 & 64.32 & 61.38 & 52.74 & 50.93 \\
31 & 94.26 & 95.90 & 96.33 & 96.03 & 93.48 & 92.80 \\
32 & 99.98 & 99.96 & 99.99 & 99.99 & 99.97 & 99.97 \\
\hline Av. & 72.34 & 73.33 & 75.48 & 73.48 & 71.89 & 70.52 \\
\hline & & & & & & \\
\hline
\end{tabular}

Table 2. Skew PAB populations; sample size $n=10,15,20,30,50,70$; estimates of power of $W$ and $T$ based on 100000 replications

\begin{tabular}{|c|r|r|r|r|r|r|}
\hline$n$ & \multicolumn{6}{|c|}{10} \\
\hline Alt. $\backslash$ Test & \multicolumn{1}{|c|}{$W$} & $\hat{T}_{10 ; c_{4}}^{(1.4,1)}$ & $\hat{T}_{10}^{(0.1,2)}$ & $\hat{T}_{10}^{(-1.45,3)}$ & $\hat{T}_{10 ; c_{1}}^{(-1.25,4)}$ & $\hat{T}_{10 ; c_{1}}^{(-1.01,5)}$ \\
\hline 36 & 32.92 & 20.27 & 19.00 & 21.08 & 26.09 & 29.50 \\
37 & 5.18 & 2.47 & 2.96 & 5.17 & 5.10 & 5.05 \\
38 & 13.13 & 5.65 & 7.56 & 12.08 & 11.08 & 10.29 \\
39 & 5.11 & 6.24 & 6.32 & 5.73 & 5.69 & 5.52 \\
40 & 13.48 & 16.08 & 15.98 & 14.13 & 14.94 & 15.78 \\
41 & 13.23 & 17.39 & 17.43 & 15.48 & 15.21 & 14.85 \\
42 & 47.27 & 44.19 & 44.22 & 44.49 & 46.80 & 46.09 \\
43 & 78.60 & 56.23 & 56.29 & 64.41 & 70.50 & 70.20 \\
44 & 8.31 & 11.34 & 11.30 & 9.89 & 9.41 & 9.24 \\
45 & 13.05 & 18.49 & 18.68 & 15.73 & 13.90 & 13.02 \\
46 & 11.80 & 16.38 & 16.50 & 14.22 & 13.35 & 12.92 \\
47 & 10.30 & 14.23 & 14.42 & 12.32 & 10.80 & 9.88 \\
\hline
\end{tabular}


Table 2 (cont.)

\begin{tabular}{|c|c|c|c|c|c|c|}
\hline$n$ & \multicolumn{6}{|c|}{10} \\
\hline Alt. $\backslash$ Test & $W$ & $\hat{T}_{10 ; c_{4}}^{(1.4,1)}$ & $\hat{T}_{10}^{(0.1,2)}$ & $\hat{T}_{10}^{(-1.45,3)}$ & $\hat{T}_{10 ; c_{1}}^{(-1.25,4)}$ & $\hat{T}_{10 ; c_{1}}^{(-1.01,5)}$ \\
\hline 48 & 40.27 & 46.04 & 46.35 & 43.67 & 40.05 & 36.39 \\
\hline 49 & 11.67 & 15.60 & 15.54 & 13.52 & 12.39 & 11.57 \\
\hline 50 & 23.74 & 28.97 & 28.59 & 25.88 & 26.23 & 26.07 \\
\hline 51 & 61.86 & 58.82 & 58.38 & 59.99 & 59.40 & 56.47 \\
\hline 52 & 27.77 & 32.89 & 33.34 & 29.98 & 26.52 & 23.67 \\
\hline 53 & 44.48 & 45.15 & 44.09 & 43.08 & 45.50 & 45.95 \\
\hline 54 & 40.06 & 40.84 & 40.93 & 40.76 & 39.03 & 36.51 \\
\hline 55 & 73.44 & 65.34 & 63.52 & 65.44 & 69.42 & 72.23 \\
\hline 56 & 89.68 & 82.02 & 80.26 & 83.27 & 86.46 & 88.20 \\
\hline 57 & 41.38 & 35.51 & 30.80 & 34.60 & 36.99 & 35.20 \\
\hline 58 & 60.65 & 61.49 & 60.25 & 59.94 & 61.98 & 62.99 \\
\hline Av. & 33.36 & 32.24 & 31.86 & 31.95 & 32.47 & 32.07 \\
\hline$n$ & \multicolumn{6}{|c|}{15} \\
\hline Alt. $\backslash$ Test & $W$ & $\hat{T}_{15}^{(-0.5,1)}$ & $\hat{T}_{15}^{(0.1,2)}$ & $\hat{T}_{15}^{(-1.43,3)}$ & $\hat{T}_{15 ; c_{1}}^{(-1.25,4)}$ & $\hat{T}_{15 ; c_{1}}^{(-0.99,5)}$ \\
\hline 36 & 53.49 & 42.29 & 24.83 & 27.64 & 38.13 & 42.63 \\
\hline 37 & 5.87 & 4.21 & 4.82 & 6.59 & 6.40 & 6.39 \\
\hline 38 & 20.89 & 11.76 & 18.27 & 20.53 & 17.12 & 15.78 \\
\hline 39 & 5.39 & 5.54 & 6.88 & 6.11 & 6.30 & 6.52 \\
\hline 40 & 21.11 & 20.64 & 21.76 & 19.36 & 22.84 & 24.18 \\
\hline 41 & 19.21 & 20.32 & 23.95 & 21.43 & 22.40 & 22.44 \\
\hline 42 & 71.82 & 70.06 & 61.97 & 64.83 & 69.89 & 69.36 \\
\hline 43 & 95.21 & 92.58 & 76.68 & 81.28 & 88.11 & 89.31 \\
\hline 44 & 11.47 & 11.90 & 14.47 & 12.44 & 12.77 & 13.20 \\
\hline 45 & 19.06 & 20.40 & 25.03 & 21.56 & 19.39 & 18.10 \\
\hline 46 & 17.87 & 18.86 & 22.28 & 19.70 & 19.81 & 19.19 \\
\hline 47 & 14.42 & 15.91 & 18.04 & 15.78 & 13.49 & 12.10 \\
\hline 48 & 61.73 & 62.86 & 64.54 & 62.68 & 57.17 & 52.90 \\
\hline 49 & 16.19 & 17.60 & 20.20 & 18.12 & 16.20 & 15.31 \\
\hline 50 & 38.79 & 39.14 & 41.63 & 38.71 & 41.03 & 41.16 \\
\hline 51 & 79.33 & 79.56 & 77.35 & 78.09 & 75.66 & 72.91 \\
\hline 52 & 42.69 & 44.71 & 44.84 & 42.93 & 36.54 & 32.68 \\
\hline 53 & 67.47 & 65.16 & 62.61 & 61.61 & 66.40 & 67.55 \\
\hline 54 & 54.98 & 55.30 & 54.89 & 54.69 & 51.60 & 48.58 \\
\hline 55 & 92.57 & 88.42 & 83.00 & 83.96 & 88.72 & 89.99 \\
\hline 56 & 98.70 & 97.33 & 94.29 & 95.42 & 97.30 & 97.81 \\
\hline 57 & 59.60 & 60.62 & 51.77 & 56.05 & 47.24 & 46.61 \\
\hline 58 & 82.48 & 81.19 & 79.78 & 79.26 & 82.61 & 83.25 \\
\hline Av. & 45.67 & 44.62 & 43.21 & 42.99 & 43.35 & 42.95 \\
\hline$n$ & \multicolumn{6}{|c|}{20} \\
\hline Alt. $\backslash$ Test & $W$ & $\hat{T}_{20}^{(-0.5,1)}$ & $\hat{T}_{20}^{(0.1,2)}$ & $\hat{T}_{20}^{(-1.45,3)}$ & $\hat{T}_{20 ; c_{1}}^{(-1.4,4)}$ & $\hat{T}_{20 ; c_{1}}^{(-0.9,5)}$ \\
\hline 36 & 72.24 & 59.36 & 30.39 & 35.82 & 55.81 & 48.34 \\
\hline 37 & 7.48 & 4.28 & 7.15 & 8.21 & 6.79 & 7.64 \\
\hline 38 & 30.33 & 13.98 & 29.87 & 28.50 & 20.24 & 22.18 \\
\hline 39 & 5.96 & 6.38 & 7.54 & 6.62 & 7.03 & 7.11 \\
\hline
\end{tabular}


Table 2 (cont.)

\begin{tabular}{|c|c|c|c|c|c|c|}
\hline$n$ & \multicolumn{6}{|c|}{20} \\
\hline Alt. \Test & $W$ & $\hat{T}_{20}^{(-0.5,1)}$ & $\hat{T}_{20}^{(0.1,2)}$ & $\hat{T}_{20}^{(-1.45,3)}$ & $\hat{T}_{20 ; c_{1}}^{(-1.4,4)}$ & $\hat{T}_{20 ; c_{1}}^{(-0.9,5)}$ \\
\hline 40 & 30.24 & 30.51 & 28.08 & 26.16 & 33.61 & 30.22 \\
\hline 41 & 26.08 & 27.60 & 30.75 & 29.01 & 29.62 & 29.83 \\
\hline 42 & 86.86 & 86.19 & 73.91 & 80.06 & 83.62 & 83.83 \\
\hline 43 & 98.65 & 98.04 & 84.14 & 91.26 & 95.68 & 95.76 \\
\hline 44 & 15.32 & 16.62 & 18.13 & 15.52 & 16.74 & 15.90 \\
\hline 45 & 24.91 & 27.40 & 30.98 & 27.64 & 22.04 & 23.09 \\
\hline 46 & 24.36 & 26.43 & 28.19 & 25.69 & 25.25 & 24.56 \\
\hline 47 & 18.35 & 21.46 & 21.19 & 18.68 & 13.57 & 14.69 \\
\hline 48 & 76.47 & 77.90 & 75.39 & 74.93 & 63.35 & 66.07 \\
\hline 49 & 20.95 & 23.33 & 24.26 & 22.45 & 18.20 & 19.18 \\
\hline 50 & 52.91 & 53.96 & 53.70 & 50.73 & 54.59 & 51.87 \\
\hline 51 & 87.92 & 88.17 & 86.34 & 87.15 & 80.78 & 83.37 \\
\hline 52 & 54.54 & 57.33 & 51.99 & 51.57 & 38.24 & 41.40 \\
\hline 53 & 83.64 & 81.05 & 76.65 & 76.11 & 82.30 & 79.21 \\
\hline 54 & 65.66 & 65.86 & 63.91 & 64.47 & 55.35 & 58.88 \\
\hline 55 & 98.40 & 96.61 & 92.41 & 93.51 & 97.02 & 95.88 \\
\hline 56 & 99.90 & 99.63 & 98.52 & 98.98 & 99.67 & 99.47 \\
\hline 57 & 72.63 & 71.35 & 66.29 & 69.51 & 67.37 & 50.38 \\
\hline 58 & 93.16 & 92.46 & 90.16 & 90.40 & 93.21 & 91.86 \\
\hline Av. & 54.22 & 53.30 & 50.87 & 51.00 & 50.44 & 49.60 \\
\hline$n$ & \multicolumn{6}{|c|}{30} \\
\hline Alt. \Test & $W$ & $\hat{T}_{30}^{(-0.5,1)}$ & $\hat{T}_{30}^{(-0.9,2)}$ & $\hat{T}_{30}^{(-1.49,3)}$ & $\begin{array}{l}\hat{T}_{30 ; c_{1}}^{(-1.3,4)} \\
\end{array}$ & $\begin{array}{l}\hat{T}_{30 ; c_{3}}^{(-0.9,5)} \\
\end{array}$ \\
\hline 36 & 92.76 & 81.90 & 86.85 & 51.63 & 67.55 & 71.44 \\
\hline 37 & 10.61 & 4.50 & 6.48 & 10.98 & 9.63 & 9.56 \\
\hline 38 & 51.76 & 19.59 & 21.45 & 42.09 & 34.00 & 34.35 \\
\hline 39 & 7.30 & 7.98 & 6.78 & 7.84 & 8.46 & 5.67 \\
\hline 40 & 49.31 & 48.50 & 43.25 & 40.75 & 47.56 & 43.31 \\
\hline 41 & 38.89 & 40.58 & 35.68 & 44.19 & 45.71 & 32.57 \\
\hline 42 & 97.84 & 97.40 & 96.82 & 94.48 & 96.43 & 92.42 \\
\hline 43 & 99.87 & 99.84 & 99.84 & 98.27 & 99.36 & 99.36 \\
\hline 44 & 23.50 & 25.34 & 19.33 & 22.60 & 24.11 & 20.03 \\
\hline 45 & 36.06 & 39.41 & 28.03 & 38.82 & 33.96 & 29.12 \\
\hline 46 & 36.70 & 39.31 & 30.04 & 37.42 & 37.86 & 33.78 \\
\hline 47 & 25.84 & 30.70 & 19.95 & 24.24 & 19.56 & 18.95 \\
\hline 48 & 91.05 & 92.21 & 87.56 & 87.47 & 81.61 & 84.53 \\
\hline 49 & 29.19 & 32.52 & 24.52 & 30.20 & 26.69 & 24.11 \\
\hline 50 & 74.96 & 74.68 & 65.75 & 70.41 & 73.38 & 72.56 \\
\hline 51 & 95.86 & 95.95 & 95.69 & 95.07 & 92.73 & 95.12 \\
\hline 52 & 71.42 & 74.45 & 67.65 & 63.52 & 53.51 & 60.75 \\
\hline 53 & 96.80 & 94.98 & 92.12 & 91.83 & 94.21 & 95.37 \\
\hline 54 & 79.30 & 79.48 & 79.20 & 77.34 & 71.83 & 77.73 \\
\hline 55 & 99.95 & 99.75 & 99.57 & 99.26 & 99.66 & 99.85 \\
\hline 56 & 100.00 & 100.00 & 99.99 & 99.96 & 99.99 & 100.00 \\
\hline 57 & 87.54 & 86.28 & 88.83 & 82.86 & 74.32 & 72.42 \\
\hline 58 & 99.18 & 98.90 & 98.14 & 98.22 & 98.77 & 98.95 \\
\hline Av. & 65.03 & 63.66 & 60.59 & 61.28 & 60.47 & 59.65 \\
\hline
\end{tabular}


Table 2 (cont.)

\begin{tabular}{|c|c|c|c|c|c|c|}
\hline$n$ & \multicolumn{6}{|c|}{50} \\
\hline Alt. $\backslash$ Test & $W$ & $\hat{T}_{50}^{(0.6,1)}$ & $\hat{T}_{50}^{(0.1,2)}$ & $\hat{T}_{50}^{(-1.47,3)}$ & $\hat{T}_{50 ; c_{3}}^{(-0.9,4)}$ & $\hat{T}_{50 ; c_{3}}^{(-0.9,5)}$ \\
\hline 36 & 99.83 & 83.66 & 55.26 & 69.19 & 87.65 & 93.14 \\
\hline 37 & 20.10 & 4.27 & 21.90 & 18.89 & 17.78 & 13.57 \\
\hline 38 & 84.00 & 33.03 & 77.73 & 68.04 & 64.57 & 53.18 \\
\hline 39 & 9.92 & 9.51 & 11.62 & 9.41 & 8.61 & 9.09 \\
\hline 40 & 79.39 & 64.98 & 65.04 & 60.06 & 68.47 & 73.99 \\
\hline 41 & 61.86 & 61.02 & 66.70 & 66.91 & 59.83 & 58.93 \\
\hline 42 & 99.97 & 99.74 & 97.64 & 99.48 & 99.67 & 99.76 \\
\hline 43 & 100.00 & 99.98 & 99.19 & 99.86 & 99.99 & 99.99 \\
\hline 44 & 41.51 & 37.93 & 41.26 & 33.51 & 37.22 & 39.34 \\
\hline 45 & 55.73 & 61.26 & 62.23 & 57.58 & 53.76 & 49.94 \\
\hline 46 & 58.91 & 59.40 & 62.30 & 55.23 & 58.14 & 59.36 \\
\hline 47 & 38.86 & 47.00 & 37.76 & 33.70 & 33.81 & 30.17 \\
\hline 48 & 98.61 & 98.99 & 96.84 & 96.81 & 97.49 & 96.70 \\
\hline 49 & 43.33 & 48.83 & 46.18 & 43.89 & 41.82 & 39.30 \\
\hline 50 & 94.81 & 91.27 & 92.16 & 88.94 & 92.67 & 94.23 \\
\hline 51 & 99.53 & 99.58 & 98.77 & 99.19 & 99.40 & 99.37 \\
\hline 52 & 88.01 & 90.24 & 78.92 & 78.82 & 82.88 & 79.98 \\
\hline 53 & 99.94 & 99.39 & 99.24 & 99.00 & 99.73 & 99.82 \\
\hline 54 & 92.57 & 92.72 & 87.72 & 90.32 & 92.10 & 91.45 \\
\hline 55 & 100.00 & 100.00 & 99.97 & 99.98 & 100.00 & 100.00 \\
\hline 56 & 100.00 & 100.00 & 100.00 & 100.00 & 100.00 & 100.00 \\
\hline 57 & 97.54 & 97.46 & 95.73 & 96.18 & 63.56 & 75.78 \\
\hline 58 & 100.00 & 99.95 & 99.94 & 99.93 & 99.98 & 99.99 \\
\hline Av. & 76.71 & 73.05 & 73.66 & 72.39 & 72.14 & 72.05 \\
\hline$n$ & \multicolumn{6}{|c|}{70} \\
\hline Alt. \Test & $W$ & $\hat{T}_{70}^{(-0.5,1)}$ & $\hat{T}_{70}^{(-0.95,2)}$ & $\hat{T}_{70}^{(-0.9,3)}$ & $\hat{T}_{70 ; c_{3}}^{(-0.95,4)}$ & $\hat{T}_{70 ; c_{3}}^{(-0.9,5)}$ \\
\hline 36 & 100.00 & 99.77 & 99.93 & 99.91 & 97.43 & 98.67 \\
\hline 37 & 32.45 & 6.70 & 18.14 & 35.35 & 18.27 & 18.18 \\
\hline 38 & 96.72 & 46.33 & 79.36 & 91.31 & 70.67 & 68.94 \\
\hline 39 & 13.30 & 13.57 & 13.33 & 9.60 & 12.43 & 12.79 \\
\hline 40 & 94.01 & 89.89 & 87.70 & 85.07 & 88.14 & 89.86 \\
\hline 41 & 78.01 & 77.93 & 76.38 & 65.89 & 78.39 & 78.13 \\
\hline 42 & 100.00 & 100.00 & 100.00 & 99.99 & 99.99 & 100.00 \\
\hline 43 & 100.00 & 100.00 & 100.00 & 100.00 & 100.00 & 100.00 \\
\hline 44 & 58.60 & 55.16 & 50.10 & 44.04 & 55.91 & 56.43 \\
\hline 45 & 70.60 & 71.67 & 68.77 & 66.86 & 69.28 & 66.63 \\
\hline 46 & 75.64 & 75.57 & 70.14 & 65.89 & 76.62 & 76.85 \\
\hline 47 & 50.03 & 56.62 & 50.28 & 45.58 & 43.86 & 41.01 \\
\hline 48 & 99.75 & 99.83 & 99.78 & 99.58 & 99.43 & 99.32 \\
\hline 49 & 55.05 & 58.08 & 54.85 & 52.70 & 53.91 & 51.90 \\
\hline 50 & 99.28 & 98.68 & 97.86 & 97.68 & 98.75 & 98.95 \\
\hline 51 & 99.93 & 99.94 & 99.94 & 99.93 & 99.92 & 99.92 \\
\hline 52 & 94.89 & 96.01 & 94.82 & 92.45 & 90.78 & 89.59 \\
\hline 53 & 100.00 & 99.99 & 99.99 & 99.99 & 99.99 & 100.00 \\
\hline 54 & 97.34 & 97.34 & 97.41 & 97.13 & 96.93 & 96.65 \\
\hline 55 & 100.00 & 100.00 & 100.00 & 100.00 & 100.00 & 100.00 \\
\hline
\end{tabular}


Table 2 (cont.)

\begin{tabular}{|c|r|r|r|r|r|r|}
\hline$n$ & \multicolumn{6}{|c|}{70} \\
\hline Alt. $\backslash$ Test & \multicolumn{1}{|c|}{$W$} & $\hat{T}_{70}^{(-0.5,1)}$ & $\hat{T}_{70}^{(-0.95,2)}$ & $\hat{T}_{70}^{(-0.9,3)}$ & $\hat{T}_{70 ; c_{3}}^{(-0.95,4)}$ & $\hat{T}_{70 ; c_{3}}^{(-0.9,5)}$ \\
\hline 56 & 100.00 & 100.00 & 100.00 & 100.00 & 100.00 & 100.00 \\
57 & 99.62 & 99.19 & 99.64 & 96.11 & 87.34 & 76.59 \\
58 & 100.00 & 100.00 & 100.00 & 100.00 & 100.00 & 100.00 \\
\hline Av. & 83.27 & 80.10 & 80.80 & 80.22 & 79.92 & 79.15 \\
\hline
\end{tabular}

Table 3. Symmetric KL and CC populations; sample size $n=10,15,20,30,50,70$; estimates of power of $W$ and $T$ based on 100000 replications

\begin{tabular}{|c|c|c|c|c|c|c|}
\hline$n$ & \multicolumn{6}{|c|}{10} \\
\hline Alt. \Test & $W$ & $\hat{T}_{10}^{(-1.25,1)}$ & $\hat{T}_{10}^{(-1.25,2)}$ & $\hat{T}_{10}^{(-1.01,3)}$ & $\hat{T}_{10}^{(-1.1,4)}$ & $\hat{T}_{10}^{(0.5,5)}$ \\
\hline 1 & 16.05 & 10.88 & 17.38 & 11.64 & 7.63 & 20.89 \\
\hline 2 & 9.74 & 7.18 & 11.89 & 7.65 & 5.42 & 15.38 \\
\hline 5 & 5.78 & 4.84 & 7.95 & 5.24 & 4.00 & 10.71 \\
\hline 15 & 8.11 & 8.92 & 8.12 & 8.89 & 8.28 & 5.81 \\
\hline 17 & 52.33 & 38.34 & 38.36 & 44.07 & 39.73 & 35.74 \\
\hline 20 & 11.23 & 12.27 & 11.44 & 12.12 & 11.16 & 7.95 \\
\hline 22 & 40.08 & 39.67 & 37.75 & 40.95 & 37.03 & 26.55 \\
\hline 25 & 19.66 & 20.80 & 20.23 & 20.52 & 19.37 & 14.72 \\
\hline 27 & 25.55 & 26.32 & 25.71 & 26.37 & 24.75 & 20.16 \\
\hline 28 & 23.40 & 24.97 & 23.11 & 24.91 & 22.91 & 15.22 \\
\hline Av. & 21.19 & 19.42 & 20.19 & 20.24 & 18.03 & 17.31 \\
\hline$n$ & \multicolumn{6}{|c|}{15} \\
\hline Alt. \Test & $W$ & $\hat{T}_{15 ; c_{4}}^{(-0.6,1)}$ & $\hat{T}_{15 ; c_{1}}^{(-1.3,2)}$ & $\hat{T}_{15}^{(-0.6,3)}$ & $\hat{T}_{15}^{(0.1,4)}$ & $\hat{T}_{15}^{(0.5,5)}$ \\
\hline 1 & 28.48 & 32.34 & 36.68 & 37.80 & 35.24 & 32.63 \\
\hline 2 & 15.98 & 21.61 & 25.13 & 26.39 & 24.25 & 22.96 \\
\hline 5 & 7.79 & 12.74 & 15.60 & 16.92 & 15.65 & 15.07 \\
\hline 15 & 9.84 & 10.08 & 9.07 & 7.87 & 7.87 & 7.38 \\
\hline 17 & 68.48 & 47.95 & 51.75 & 57.00 & 59.99 & 62.74 \\
\hline 20 & 15.39 & 15.80 & 14.83 & 12.95 & 12.31 & 11.75 \\
\hline 22 & 57.72 & 55.35 & 54.43 & 51.82 & 50.96 & 50.23 \\
\hline 25 & 28.40 & 29.05 & 27.99 & 25.29 & 25.20 & 23.84 \\
\hline 27 & 36.16 & 36.88 & 35.91 & 33.59 & 32.98 & 32.33 \\
\hline 28 & 33.45 & 33.58 & 32.24 & 29.78 & 28.82 & 27.93 \\
\hline Av. & 30.17 & 29.54 & 30.36 & 29.94 & 29.33 & 28.69 \\
\hline$n$ & \multicolumn{6}{|c|}{20} \\
\hline Alt. \Test & $W$ & $\hat{T}_{20 ; c_{4}}^{(-0.6,1)}$ & $\hat{T}_{20 ; c_{1}}^{(-1.25,2)}$ & $\hat{T}_{20}^{(-0.6,3)}$ & $\hat{T}_{20}^{(0.1,4)}$ & $\hat{T}_{20}^{(0.7,5)}$ \\
\hline 1 & 44.10 & 46.75 & 50.44 & 51.95 & 48.92 & 45.70 \\
\hline 2 & 24.95 & 31.28 & 35.54 & 36.47 & 34.16 & 31.49 \\
\hline 5 & 11.52 & 17.70 & 21.52 & 22.34 & 21.15 & 19.54 \\
\hline 15 & 11.61 & 12.40 & 11.18 & 9.80 & 9.74 & 8.88 \\
\hline 17 & 80.59 & 59.91 & 65.17 & 73.52 & 76.67 & 79.62 \\
\hline 20 & 19.26 & 20.46 & 18.95 & 16.42 & 16.04 & 14.49 \\
\hline 22 & 70.62 & 70.81 & 69.54 & 67.85 & 67.82 & 66.21 \\
\hline
\end{tabular}


Table 3 (cont.)

\begin{tabular}{|c|c|c|c|c|c|c|}
\hline$n$ & \multicolumn{6}{|c|}{20} \\
\hline Alt. $\backslash$ Test & $W$ & $\hat{T}_{20 ; c_{4}}^{(-0.6,1)}$ & $\hat{T}_{20 ; c_{1}}^{(-1.25,2)}$ & $\hat{T}_{20}^{(-0.6,3)}$ & $\hat{T}_{20}^{(0.1,4)}$ & $\hat{T}_{20}^{(0.7,5)}$ \\
\hline 25 & 36.09 & 37.11 & 35.56 & 32.87 & 32.44 & 30.45 \\
\hline 27 & 45.16 & 46.10 & 44.58 & 42.55 & 42.46 & 40.58 \\
\hline 28 & 42.44 & 44.76 & 43.05 & 40.85 & 41.15 & 39.17 \\
\hline Av. & 38.63 & 38.73 & 39.55 & 39.46 & 39.05 & 37.61 \\
\hline$n$ & \multicolumn{6}{|c|}{30} \\
\hline Alt. $\backslash$ Test & $W$ & $\begin{array}{l}\hat{T}_{30 ; c_{4}}^{(-0.6,1)} \\
\end{array}$ & $\hat{T}_{30 ; c_{1}}^{(-1.35,2)}$ & $\hat{T}_{30}^{(-0.6,3)}$ & $\hat{T}_{30}^{(0.1,4)}$ & $\hat{T}_{30}^{(0.5,5)}$ \\
\hline 1 & 73.01 & 73.02 & 75.24 & 74.69 & 69.91 & 66.34 \\
\hline 2 & 47.46 & 55.02 & 57.44 & 56.94 & 50.83 & 48.29 \\
\hline 5 & 21.19 & 32.15 & 35.67 & 35.77 & 31.12 & 29.49 \\
\hline 15 & 14.80 & 16.61 & 14.94 & 13.15 & 12.66 & 11.69 \\
\hline 17 & 94.08 & 76.87 & 83.45 & 90.90 & 92.71 & 94.74 \\
\hline 20 & 26.66 & 27.54 & 25.89 & 22.70 & 21.24 & 19.59 \\
\hline 22 & 86.31 & 87.70 & 87.55 & 86.08 & 85.67 & 84.94 \\
\hline 25 & 48.66 & 49.10 & 48.20 & 45.44 & 44.14 & 42.12 \\
\hline 27 & 59.21 & 58.16 & 58.75 & 56.34 & 55.40 & 54.47 \\
\hline 28 & 57.01 & 60.94 & 60.48 & 59.16 & 58.84 & 57.24 \\
\hline Av. & 52.84 & 53.71 & 54.76 & 54.12 & 52.25 & 50.89 \\
\hline$n$ & \multicolumn{6}{|c|}{50} \\
\hline Alt. \Test & $W$ & $\hat{T}_{50 ; c_{2}}^{(-0.7,1)}$ & $\hat{T}_{50 ; c_{1}}^{(-1.35,2)}$ & $\hat{T}_{50}^{(-0.6,3)}$ & $\hat{T}_{50}^{(-0.1,4)}$ & $\hat{T}_{50 ; c_{3}}^{(0.1,5)}$ \\
\hline 1 & 97.14 & 94.80 & 95.94 & 95.09 & 91.97 & 90.07 \\
\hline 2 & 84.01 & 83.47 & 86.80 & 83.76 & 77.68 & 83.76 \\
\hline 5 & 47.77 & 56.85 & 64.22 & 59.95 & 52.73 & 66.67 \\
\hline 15 & 19.28 & 25.20 & 21.76 & 19.59 & 18.56 & 11.87 \\
\hline 17 & 99.68 & 90.50 & 97.17 & 99.20 & 99.56 & 98.63 \\
\hline 20 & 37.77 & 42.05 & 36.89 & 33.38 & 31.20 & 24.46 \\
\hline 22 & 97.14 & 97.94 & 97.92 & 97.53 & 97.17 & 94.10 \\
\hline 25 & 66.23 & 68.49 & 65.10 & 62.70 & 60.70 & 54.37 \\
\hline 27 & 76.59 & 78.30 & 76.00 & 74.62 & 73.25 & 69.03 \\
\hline 28 & 76.54 & 81.40 & 81.77 & 80.93 & 80.11 & 68.17 \\
\hline Av. & 70.22 & 71.90 & 72.36 & 70.67 & 68.29 & 66.11 \\
\hline$n$ & \multicolumn{6}{|c|}{70} \\
\hline Alt. \Test & $W$ & $\hat{T}_{70}^{(-1.25,1)}$ & $\hat{T}_{70 ; c_{1}}^{(-1.3,2)}$ & $\hat{T}_{70}^{(-0.6,3)}$ & $\hat{T}_{70 ; c_{3}}^{(-0.3,4)}$ & $\hat{T}_{70 ; c_{3}}^{(-0.1,5)}$ \\
\hline 1 & 99.89 & 98.33 & 99.55 & 99.30 & 95.94 & 92.87 \\
\hline 2 & 97.37 & 89.74 & 97.03 & 95.12 & 94.96 & 92.42 \\
\hline 5 & 73.22 & 59.84 & 83.36 & 77.73 & 84.91 & 82.10 \\
\hline 15 & 24.30 & 33.06 & 27.97 & 25.32 & 18.78 & 17.29 \\
\hline 17 & 99.98 & 96.83 & 99.67 & 99.94 & 99.86 & 99.78 \\
\hline 20 & 47.17 & 53.44 & 45.23 & 42.16 & 37.02 & 35.17 \\
\hline 22 & 99.45 & 99.68 & 99.61 & 99.48 & 99.16 & 99.03 \\
\hline 25 & 77.47 & 80.42 & 76.10 & 74.19 & 70.72 & 69.51 \\
\hline 27 & 86.67 & 88.40 & 86.09 & 84.94 & 82.77 & 82.27 \\
\hline 28 & 87.61 & 91.60 & 91.91 & 91.47 & 86.02 & 84.94 \\
\hline Av. & 79.31 & 79.13 & 80.65 & 78.97 & 77.01 & 75.54 \\
\hline
\end{tabular}


Table 4. Skew KL populations; sample size $n=10,15,20,30,50,70$; estimates of power of $W$ and $T$ based on 100000 replications

\begin{tabular}{|c|c|c|c|c|c|c|}
\hline$n$ & \multicolumn{6}{|c|}{10} \\
\hline Alt. $\backslash$ Test & $W$ & $\hat{T}_{10 ; c_{4}}^{(1.35,1)}$ & $\hat{T}_{10}^{(-0.1,2)}$ & $\hat{T}_{10}^{(-1.45,3)}$ & $\hat{T}_{10 ; c_{1}}^{(-1.25,4)}$ & $\hat{T}_{10 ; c_{1}}^{(-1.01,5)}$ \\
\hline 36 & 32.92 & 20.25 & 20.62 & 21.08 & 26.09 & 29.50 \\
\hline 40 & 13.48 & 15.66 & 14.98 & 14.13 & 14.94 & 15.78 \\
\hline 41 & 13.23 & 16.92 & 16.00 & 15.48 & 15.21 & 14.85 \\
\hline 44 & 8.31 & 10.92 & 10.03 & 9.89 & 9.41 & 9.24 \\
\hline 45 & 13.05 & 17.84 & 16.42 & 15.73 & 13.90 & 13.02 \\
\hline 46 & 11.80 & 16.09 & 14.69 & 14.22 & 13.35 & 12.92 \\
\hline 47 & 10.30 & 13.89 & 12.45 & 12.32 & 10.80 & 9.88 \\
\hline 48 & 40.27 & 45.38 & 43.64 & 43.67 & 40.05 & 36.39 \\
\hline 49 & 11.67 & 15.23 & 14.01 & 13.52 & 12.39 & 11.57 \\
\hline 50 & 23.74 & 28.62 & 27.08 & 25.88 & 26.23 & 26.07 \\
\hline 52 & 27.77 & 32.28 & 30.78 & 29.98 & 26.52 & 23.67 \\
\hline 54 & 40.06 & 40.91 & 40.45 & 40.76 & 39.03 & 36.51 \\
\hline 57 & 41.38 & 36.18 & 38.63 & 34.60 & 36.99 & 35.20 \\
\hline 58 & 60.65 & 60.58 & 60.38 & 59.94 & 61.98 & 62.99 \\
\hline Av. & 24.90 & 26.48 & 25.73 & 25.09 & 24.78 & 24.11 \\
\hline$n$ & \multicolumn{6}{|c|}{15} \\
\hline Alt. \Test & $W$ & $\hat{T}_{15}^{(1.25,1)}$ & $\hat{T}_{15}^{(0.1,2)}$ & $\hat{T}_{15 ; c_{3}}^{(1.4,3)}$ & $\hat{T}_{15 ; c_{1}}^{(-1.25,4)}$ & $\hat{T}_{15 ; c_{1}}^{(-0.95,5)}$ \\
\hline 36 & 53.49 & 21.71 & 24.83 & 28.97 & 38.13 & 40.74 \\
\hline 40 & 21.11 & 20.16 & 21.76 & 26.98 & 22.84 & 23.65 \\
\hline 41 & 19.21 & 22.07 & 23.95 & 27.75 & 22.40 & 22.24 \\
\hline 44 & 11.47 & 14.73 & 14.47 & 17.86 & 12.77 & 12.79 \\
\hline 45 & 19.06 & 26.60 & 25.03 & 26.00 & 19.39 & 18.37 \\
\hline 46 & 17.87 & 22.89 & 22.28 & 25.90 & 19.81 & 19.15 \\
\hline 47 & 14.42 & 20.70 & 18.04 & 17.88 & 13.49 & 12.54 \\
\hline 48 & 61.73 & 66.21 & 64.54 & 56.20 & 57.17 & 53.71 \\
\hline 49 & 16.19 & 21.62 & 20.20 & 21.07 & 16.20 & 15.22 \\
\hline 50 & 38.79 & 41.35 & 41.63 & 45.61 & 41.03 & 40.53 \\
\hline 52 & 42.69 & 48.82 & 44.84 & 36.80 & 36.54 & 33.43 \\
\hline 54 & 54.98 & 55.88 & 54.89 & 43.33 & 51.60 & 49.11 \\
\hline 57 & 59.60 & 55.11 & 51.77 & 33.33 & 47.24 & 44.38 \\
\hline 58 & 82.48 & 78.49 & 79.78 & 76.55 & 82.61 & 82.94 \\
\hline Av. & 36.65 & 36.88 & 36.29 & 34.59 & 34.37 & 33.49 \\
\hline$n$ & \multicolumn{6}{|c|}{20} \\
\hline Alt. $\backslash$ Test & $W$ & $\hat{T}_{20}^{(-0.5,1)}$ & $\begin{array}{l}\hat{T}_{20 ; c_{3}}^{(0.1,2)} \\
\end{array}$ & $\hat{T}_{20 ; c_{3}}^{(1.2,3)}$ & $\hat{T}_{20 ; c_{1}}^{(-1.4,4)}$ & $\hat{T}_{20 ; c_{1}}^{(-0.9,5)}$ \\
\hline 36 & 72.24 & 59.36 & 33.93 & 35.07 & 55.81 & 48.34 \\
\hline 40 & 30.24 & 30.51 & 35.92 & 35.33 & 33.61 & 30.22 \\
\hline 41 & 26.08 & 27.60 & 32.55 & 35.91 & 29.62 & 29.83 \\
\hline 44 & 15.32 & 16.62 & 23.43 & 22.50 & 16.74 & 15.90 \\
\hline 45 & 24.91 & 27.40 & 33.16 & 33.36 & 22.04 & 23.09 \\
\hline 46 & 24.36 & 26.43 & 34.25 & 33.34 & 25.25 & 24.56 \\
\hline 47 & 18.35 & 21.46 & 23.39 & 21.44 & 13.57 & 14.69 \\
\hline 48 & 76.47 & 77.90 & 71.58 & 68.30 & 63.35 & 66.07 \\
\hline
\end{tabular}


New normality tests

Table 4 (cont.)

\begin{tabular}{|c|c|c|c|c|c|c|}
\hline$n$ & \multicolumn{6}{|c|}{20} \\
\hline Alt. \Test & $W$ & $\hat{T}_{20}^{(-0.5,1)}$ & $\hat{T}_{20 ; c_{3}}^{(0.1,2)}$ & $\hat{T}_{20 ; c_{3}}^{(1.2,3)}$ & $\hat{T}_{20 ; c_{1}}^{(-1.4,4)}$ & $\hat{T}_{20 ; c_{1}}^{(-0.9,5)}$ \\
\hline 49 & 20.95 & 23.33 & 27.03 & 25.67 & 18.20 & 19.18 \\
\hline 50 & 52.91 & 53.96 & 61.31 & 59.08 & 54.59 & 51.87 \\
\hline 52 & 54.54 & 57.33 & 51.25 & 44.59 & 38.24 & 41.40 \\
\hline 54 & 65.66 & 65.86 & 60.39 & 51.12 & 55.35 & 58.88 \\
\hline 57 & 72.63 & 71.35 & 62.49 & 52.50 & 67.37 & 50.38 \\
\hline 58 & 93.16 & 92.46 & 89.31 & 85.59 & 93.21 & 91.86 \\
\hline Av. & 46.27 & 46.54 & 45.71 & 43.13 & 41.92 & 40.45 \\
\hline$n$ & \multicolumn{6}{|c|}{30} \\
\hline Alt. $\backslash$ Test & $W$ & $\hat{T}_{30}^{(-0.5,1)}$ & $\hat{T}_{30 ; c_{3}}^{(0.1,2)}$ & $\hat{T}_{30 ; c_{3}}^{(1.2,3)}$ & $\hat{T}_{30 ; c_{1}}^{(-1.35,4)}$ & $\hat{T}_{30 ; c_{3}}^{(-0.95,5)}$ \\
\hline 36 & 92.76 & 81.90 & 44.70 & 38.98 & 70.76 & 72.00 \\
\hline 40 & 49.31 & 48.50 & 50.90 & 46.15 & 49.05 & 42.34 \\
\hline 41 & 38.89 & 40.58 & 47.01 & 49.38 & 45.56 & 31.08 \\
\hline 44 & 23.50 & 25.34 & 32.36 & 29.59 & 24.50 & 19.59 \\
\hline 45 & 36.06 & 39.41 & 45.42 & 46.56 & 32.62 & 27.63 \\
\hline 46 & 36.70 & 39.31 & 48.15 & 45.66 & 37.95 & 32.98 \\
\hline 47 & 25.84 & 30.70 & 29.54 & 29.03 & 18.64 & 17.87 \\
\hline 48 & 91.05 & 92.21 & 86.88 & 85.41 & 80.11 & 83.22 \\
\hline 49 & 29.19 & 32.52 & 35.46 & 35.20 & 25.63 & 23.19 \\
\hline 50 & 74.96 & 74.68 & 78.86 & 74.91 & 73.99 & 72.33 \\
\hline 52 & 71.42 & 74.45 & 63.83 & 60.20 & 51.77 & 59.73 \\
\hline 54 & 79.30 & 79.48 & 74.26 & 70.11 & 70.05 & 77.21 \\
\hline 57 & 87.54 & 86.28 & 82.30 & 73.61 & 78.71 & 88.23 \\
\hline 58 & 99.18 & 98.90 & 95.59 & 93.85 & 98.89 & 98.97 \\
\hline Av. & 59.69 & 60.30 & 58.23 & 55.62 & 54.16 & 53.31 \\
\hline$n$ & \multicolumn{6}{|c|}{50} \\
\hline Alt. $\backslash$ Test & $W$ & $\hat{T}_{50}^{(-0.5,1)}$ & $\hat{T}_{50 ; c_{3}}^{(0.1,2)}$ & $\hat{T}_{50}^{(-1.47,3)}$ & $\hat{T}_{50 ; c_{3}}^{(-0.9,4)}$ & $\hat{T}_{50 ; c_{3}}^{(-0.9,5)}$ \\
\hline 36 & 99.83 & 97.46 & 62.31 & 69.19 & 87.65 & 93.14 \\
\hline 40 & 79.39 & 75.01 & 72.95 & 60.06 & 68.47 & 73.99 \\
\hline 41 & 61.86 & 61.97 & 69.66 & 66.91 & 59.83 & 58.93 \\
\hline 44 & 41.51 & 40.91 & 48.13 & 33.51 & 37.22 & 39.34 \\
\hline 45 & 55.73 & 57.61 & 64.21 & 57.58 & 53.76 & 49.94 \\
\hline 46 & 58.91 & 60.25 & 68.16 & 55.23 & 58.14 & 59.36 \\
\hline 47 & 38.86 & 44.88 & 40.22 & 33.70 & 33.81 & 30.17 \\
\hline 48 & 98.61 & 98.93 & 96.64 & 96.81 & 97.49 & 96.70 \\
\hline 49 & 43.33 & 46.82 & 49.32 & 43.89 & 41.82 & 39.30 \\
\hline 50 & 94.81 & 93.58 & 94.25 & 88.94 & 92.67 & 94.23 \\
\hline 52 & 88.01 & 90.23 & 78.86 & 78.82 & 82.88 & 79.98 \\
\hline 54 & 92.57 & 92.59 & 88.00 & 90.32 & 92.10 & 91.45 \\
\hline 57 & 97.54 & 96.85 & 95.93 & 96.18 & 63.56 & 75.78 \\
\hline 58 & 100.00 & 99.98 & 97.01 & 99.93 & 99.98 & 99.99 \\
\hline Av. & 75.07 & 75.50 & 73.26 & 69.36 & 69.24 & 70.16 \\
\hline
\end{tabular}


Table 4 (cont.)

\begin{tabular}{|c|r|r|c|c|c|c|}
\hline$n$ & \multicolumn{6}{|c|}{70} \\
\hline Alt. $\backslash$ Test & \multicolumn{1}{|c|}{$W$} & $\hat{T}_{70}^{(-0.5,1)}$ & $\hat{T}_{70}^{(-0.95,2)}$ & $\hat{T}_{70 ; c_{3}}^{(-0.99,3)}$ & $\hat{T}_{70 ; c_{3}}^{(-0.95,4)}$ & $\hat{T}_{70 ; c_{3}}^{(-0.95,5)}$ \\
\hline 36 & 100.00 & 99.77 & 99.93 & 94.10 & 97.43 & 98.94 \\
40 & 94.01 & 89.89 & 87.70 & 83.78 & 88.14 & 91.08 \\
41 & 78.01 & 77.93 & 76.38 & 77.07 & 78.39 & 77.69 \\
44 & 58.60 & 55.16 & 50.10 & 52.01 & 55.91 & 57.81 \\
45 & 70.60 & 71.67 & 68.77 & 71.35 & 69.28 & 65.52 \\
46 & 75.64 & 75.57 & 70.14 & 75.03 & 76.62 & 77.52 \\
47 & 50.03 & 56.62 & 50.28 & 46.79 & 43.86 & 39.83 \\
48 & 99.75 & 99.83 & 99.78 & 99.57 & 99.43 & 99.18 \\
49 & 55.05 & 58.08 & 54.85 & 54.92 & 53.91 & 50.39 \\
50 & 99.28 & 98.68 & 97.86 & 98.26 & 98.75 & 99.09 \\
52 & 94.89 & 96.01 & 94.82 & 92.27 & 90.78 & 89.11 \\
54 & 97.34 & 97.34 & 97.41 & 97.13 & 96.93 & 96.49 \\
57 & 99.62 & 99.19 & 99.64 & 98.49 & 87.34 & 93.02 \\
58 & 100.00 & 100.00 & 100.00 & 100.00 & 100.00 & 100.00 \\
\hline Av. & 83.77 & 83.98 & 81.98 & 81.48 & 81.20 & 81.12 \\
\hline \multicolumn{7}{|c|}{} \\
\hline
\end{tabular}

Table 5. Skew CC populations; sample size $n=10,15,20,30,50,70$; estimates of power of $W$ and $T$ based on 100000 replications

\begin{tabular}{|c|r|c|c|c|c|c|}
\hline$n$ & \multicolumn{5}{|c|}{10} \\
\hline Alt. $\backslash$ Test & \multicolumn{1}{|c|}{$W$} & $\hat{T}_{10 ; c_{4}}^{(1.4,1)}$ & $\hat{T}_{10}^{(0.1,2)}$ & $\hat{T}_{10}^{(-1.45,3)}$ & $\hat{T}_{10 ; c_{1}}^{(-1.25,4)}$ & $\hat{T}_{10 ; c_{1}}^{(-1.01,5)}$ \\
\hline 40 & 13.48 & 16.08 & 15.98 & 14.13 & 14.94 & 15.78 \\
41 & 13.23 & 17.39 & 17.43 & 15.48 & 15.21 & 14.85 \\
44 & 8.31 & 11.34 & 11.30 & 9.89 & 9.41 & 9.24 \\
45 & 13.05 & 18.49 & 18.68 & 15.73 & 13.90 & 13.02 \\
46 & 11.80 & 16.38 & 16.50 & 14.22 & 13.35 & 12.92 \\
47 & 10.30 & 14.23 & 14.42 & 12.32 & 10.80 & 9.88 \\
48 & 40.27 & 46.04 & 46.35 & 43.67 & 40.05 & 36.39 \\
49 & 11.67 & 15.60 & 15.54 & 13.52 & 12.39 & 11.57 \\
50 & 23.74 & 28.97 & 28.59 & 25.88 & 26.23 & 26.07 \\
52 & 27.77 & 32.89 & 33.34 & 29.98 & 26.52 & 23.67 \\
54 & 40.06 & 40.84 & 40.93 & 40.76 & 39.03 & 36.51 \\
57 & 41.38 & 35.51 & 30.80 & 34.60 & 36.99 & 35.20 \\
58 & 60.65 & 61.49 & 60.25 & 59.94 & 61.98 & 62.99 \\
\hline Av. & 24.29 & 27.33 & 26.93 & 25.39 & 24.68 & 23.70 \\
\hline \hline$n$ & \multicolumn{5}{|c|}{15} \\
\hline Alt. $\backslash$ Test & $W$ & $\hat{T}_{15}^{(1.4,1)}$ & $\hat{T}_{15 ; c_{3}}^{(0.3,2}$ & $\hat{T}_{15}^{(1.45,3)}$ & $\hat{T}_{15 ; c_{1}}^{(-1.25,4)}$ & $\hat{T}_{15 ; c_{1}}^{(-0.95,5)}$ \\
\hline 40 & 21.11 & 20.21 & 24.55 & 20.37 & 22.84 & 23.65 \\
41 & 19.21 & 22.32 & 24.49 & 22.01 & 22.40 & 22.24 \\
44 & 11.47 & 15.19 & 17.95 & 12.76 & 12.77 & 12.79 \\
45 & 19.06 & 27.18 & 27.36 & 21.82 & 19.39 & 18.37 \\
46 & 17.87 & 23.43 & 26.57 & 20.13 & 19.81 & 19.15 \\
\hline
\end{tabular}


Table 5 (cont.)

\begin{tabular}{|c|c|c|c|c|c|c|}
\hline$n$ & \multicolumn{6}{|c|}{15} \\
\hline Alt. \Test & $W$ & $\hat{T}_{15}^{(1.4,1)}$ & $\hat{T}_{15 ; c_{3}}^{(0.3,2)}$ & $\hat{T}_{15}^{(1.45,3)}$ & $\hat{T}_{15 ; c_{1}}^{(-1.25,4)}$ & $\hat{T}_{15 ; c_{1}}^{(-0.95,5)}$ \\
\hline 47 & 14.42 & 21.24 & 21.16 & 15.89 & 13.49 & 12.54 \\
\hline 48 & 61.73 & 66.19 & 60.13 & 63.00 & 57.17 & 53.71 \\
\hline 49 & 16.19 & 22.32 & 23.08 & 18.15 & 16.20 & 15.22 \\
\hline 50 & 38.79 & 41.05 & 45.90 & 39.56 & 41.03 & 40.53 \\
\hline 52 & 42.69 & 49.19 & 45.68 & 42.65 & 36.54 & 33.43 \\
\hline 54 & 54.98 & 55.52 & 53.04 & 54.83 & 51.60 & 49.11 \\
\hline 57 & 59.60 & 53.47 & 39.61 & 55.50 & 47.24 & 44.38 \\
\hline 58 & 82.48 & 77.92 & 80.06 & 79.85 & 82.61 & 82.94 \\
\hline Av. & 35.35 & 38.09 & 37.66 & 35.89 & 34.08 & 32.93 \\
\hline$n$ & \multicolumn{6}{|c|}{20} \\
\hline Alt. \Test & $W$ & $\hat{T}_{20}^{(1.35,1)}$ & $\hat{T}_{20 ; c_{3}}^{(0.35,2)}$ & $\hat{T}_{20 ; c_{3}}^{(1.4,3)}$ & $\hat{T}_{20 ; c_{1}}^{(-1.2,4)}$ & $\hat{T}_{20 ; c_{1}}^{(-0.9,5)}$ \\
\hline 40 & 30.24 & 25.32 & 29.59 & 32.27 & 29.36 & 30.22 \\
\hline 41 & 26.08 & 27.34 & 29.65 & 34.24 & 30.28 & 29.83 \\
\hline 44 & 15.32 & 18.30 & 21.63 & 21.46 & 16.04 & 15.90 \\
\hline 45 & 24.91 & 34.12 & 34.70 & 34.55 & 24.94 & 23.09 \\
\hline 46 & 24.36 & 29.66 & 32.91 & 32.33 & 25.20 & 24.56 \\
\hline 47 & 18.35 & 26.48 & 25.80 & 22.95 & 15.88 & 14.69 \\
\hline 48 & 76.47 & 79.88 & 73.74 & 71.03 & 70.23 & 66.07 \\
\hline 49 & 20.95 & 27.52 & 28.78 & 26.86 & 20.49 & 19.18 \\
\hline 50 & 52.91 & 52.02 & 56.42 & 56.85 & 52.19 & 51.87 \\
\hline 52 & 54.54 & 60.47 & 56.67 & 48.90 & 45.30 & 41.40 \\
\hline 54 & 65.66 & 65.73 & 64.32 & 57.86 & 61.83 & 58.88 \\
\hline 57 & 72.63 & 67.51 & 53.23 & 46.80 & 48.96 & 50.38 \\
\hline 58 & 93.16 & 88.87 & 89.63 & 87.19 & 91.69 & 91.86 \\
\hline Av. & 44.28 & 46.40 & 45.93 & 44.10 & 40.95 & 39.84 \\
\hline$n$ & \multicolumn{6}{|c|}{30} \\
\hline Alt. \Test & $W$ & $\hat{T}_{30}^{(-0.5,1)}$ & $\hat{T}_{30 ; c_{3}}^{(0.1,2)}$ & $\hat{T}_{30 ; c_{3}}^{(1.2,3)}$ & $\hat{T}_{30 ; c_{1}}^{(-1.3,4)}$ & $\hat{T}_{30 ; c_{3}}^{(-0.95,5)}$ \\
\hline 40 & 49.31 & 48.50 & 50.90 & 46.15 & 47.56 & 42.34 \\
\hline 41 & 38.89 & 40.58 & 47.01 & 49.38 & 45.71 & 31.08 \\
\hline 44 & 23.50 & 25.34 & 32.36 & 29.59 & 24.11 & 19.59 \\
\hline 45 & 36.06 & 39.41 & 45.42 & 46.56 & 33.96 & 27.63 \\
\hline 46 & 36.70 & 39.31 & 48.15 & 45.66 & 37.86 & 32.98 \\
\hline 47 & 25.84 & 30.70 & 29.54 & 29.03 & 19.56 & 17.87 \\
\hline 48 & 91.05 & 92.21 & 86.88 & 85.41 & 81.61 & 83.22 \\
\hline 49 & 29.19 & 32.52 & 35.46 & 35.20 & 26.69 & 23.19 \\
\hline 50 & 74.96 & 74.68 & 78.86 & 74.91 & 73.38 & 72.33 \\
\hline 52 & 71.42 & 74.45 & 63.83 & 60.20 & 53.51 & 59.73 \\
\hline 54 & 79.30 & 79.48 & 74.26 & 70.11 & 71.83 & 77.21 \\
\hline 57 & 87.54 & 86.28 & 82.30 & 73.61 & 74.32 & 88.23 \\
\hline 58 & 99.18 & 98.90 & 95.59 & 93.85 & 98.77 & 98.97 \\
\hline Av. & 57.15 & 58.64 & 59.27 & 56.90 & 52.99 & 51.87 \\
\hline
\end{tabular}


Table 5 (cont.)

\begin{tabular}{|c|r|r|r|r|r|r|}
\hline$n$ & \multicolumn{5}{|c|}{50} \\
\hline Alt. $\backslash$ Test & \multicolumn{1}{|c|}{$W$} & $\hat{T}_{50}^{(0.9,1)}$ & $\hat{T}_{50 ; c_{3}}^{(0.2,2)}$ & $\hat{T}_{50}^{(-1.47,3)}$ & $\hat{T}_{50 ; c_{3}}^{(-0.9,4)}$ & $\hat{T}_{50 ; c_{3}}^{(-0.9)}$ \\
\hline 40 & 79.39 & 62.77 & 68.02 & 60.06 & 68.47 & 73.99 \\
41 & 61.86 & 62.60 & 67.53 & 66.91 & 59.83 & 58.93 \\
44 & 41.51 & 39.17 & 46.13 & 33.51 & 37.22 & 39.34 \\
45 & 55.73 & 64.91 & 66.66 & 57.58 & 53.76 & 49.94 \\
46 & 58.91 & 60.93 & 67.05 & 55.23 & 58.14 & 59.36 \\
47 & 38.86 & 49.10 & 43.60 & 33.70 & 33.81 & 30.17 \\
48 & 98.61 & 99.09 & 97.43 & 96.81 & 97.49 & 96.70 \\
49 & 43.33 & 50.56 & 51.33 & 43.89 & 41.82 & 39.30 \\
50 & 94.81 & 91.05 & 93.08 & 88.94 & 92.67 & 94.23 \\
52 & 88.01 & 90.58 & 82.34 & 78.82 & 82.88 & 79.98 \\
54 & 92.57 & 92.77 & 90.39 & 90.32 & 92.10 & 91.45 \\
57 & 97.54 & 97.13 & 94.95 & 96.18 & 63.56 & 75.78 \\
58 & 100.00 & 99.96 & 98.97 & 99.93 & 99.98 & 99.99 \\
\hline Av. & 73.16 & 73.89 & 74.42 & 69.38 & 67.83 & 68.40 \\
\hline \hline$n$ & \multicolumn{7}{|c|}{70} & & \\
\hline Alt. $\backslash$ Test & $W$ & $\hat{T}_{70}^{(-0.5,1)}$ & $\hat{T}_{70}^{(-0.95,2)}$ & $\hat{T}_{70 ; c_{3}}^{(1.03)}$ & $\hat{T}_{70 ; c_{3}}^{(-0.95,4)}$ & $\hat{T}_{70 ; c_{3}}^{(-0.95,5)}$ \\
\hline 40 & 94.01 & 89.89 & 87.70 & 82.06 & 88.14 & 91.08 \\
41 & 78.01 & 77.93 & 76.38 & 86.82 & 78.39 & 77.69 \\
44 & 58.60 & 55.16 & 50.10 & 56.84 & 55.91 & 57.81 \\
45 & 70.60 & 71.67 & 68.77 & 76.50 & 69.28 & 65.52 \\
46 & 75.64 & 75.57 & 70.14 & 78.28 & 76.62 & 77.52 \\
47 & 50.03 & 56.62 & 50.28 & 46.87 & 43.86 & 39.83 \\
48 & 99.75 & 99.83 & 99.78 & 98.63 & 99.43 & 99.18 \\
49 & 55.05 & 58.08 & 54.85 & 59.86 & 53.91 & 50.39 \\
50 & 99.28 & 98.68 & 97.86 & 97.86 & 98.75 & 99.09 \\
52 & 94.89 & 96.01 & 94.82 & 83.79 & 90.78 & 89.11 \\
54 & 97.34 & 97.34 & 97.41 & 91.02 & 96.93 & 96.49 \\
57 & 99.62 & 99.19 & 99.64 & 98.75 & 87.34 & 93.02 \\
58 & 100.00 & 100.00 & 100.00 & 94.80 & 100.00 & 100.00 \\
\hline Av. & 82.52 & 82.77 & 80.59 & 80.93 & 79.95 & 79.75 \\
\hline
\end{tabular}

Table 6. Symmetric MS populations; sample size $n=10,15,20,30,50,70$; estimates of power of $W$ and $T$ based on 100000 replications

\begin{tabular}{|c|r|r|r|r|r|r|}
\hline$n$ & \multicolumn{6}{|c|}{10} \\
\hline Alt. $\backslash$ Test & \multicolumn{1}{|c|}{$W$} & $\hat{T}_{10}^{(-1.25,1)}$ & $\hat{T}_{10}^{(-1.25,2)}$ & $\hat{T}_{10}^{(-1.01,3)}$ & $\hat{T}_{10}^{(-1.01,4)}$ & $\hat{T}_{10}^{(-0.99,5)}$ \\
\hline 1 & 16.05 & 10.88 & 17.38 & 11.64 & 8.01 & 6.92 \\
4 & 6.87 & 5.42 & 8.90 & 5.84 & 4.49 & 4.12 \\
7 & 4.23 & 3.52 & 5.32 & 3.85 & 3.51 & 3.40 \\
15 & 8.11 & 8.92 & 8.12 & 8.89 & 8.53 & 8.11 \\
18 & 15.32 & 16.65 & 15.00 & 16.71 & 15.56 & 14.55 \\
21 & 15.04 & 17.05 & 15.69 & 16.87 & 15.48 & 14.44 \\
24 & 30.01 & 31.18 & 29.95 & 31.45 & 28.97 & 26.81 \\
27 & 25.55 & 26.32 & 25.71 & 26.37 & 24.92 & 23.74 \\
\hline
\end{tabular}


Table 6 (cont.)

\begin{tabular}{|c|c|c|c|c|c|c|}
\hline$n$ & \multicolumn{6}{|c|}{10} \\
\hline Alt. \Test & $W$ & $\hat{T}_{10}^{(-1.25,1)}$ & $\hat{T}_{10}^{(-1.25,2)}$ & $\hat{T}_{10}^{(-1.01,3)}$ & $\hat{T}_{10}^{(-1.01,4)}$ & $\hat{T}_{10}^{(-0.99,5)}$ \\
\hline 30 & 13.69 & 15.38 & 13.97 & 15.00 & 14.41 & 13.19 \\
\hline 32 & 59.19 & 57.86 & 56.06 & 59.22 & 55.34 & 52.65 \\
\hline Av. & 19.41 & 19.32 & 19.61 & 19.58 & 17.92 & 16.79 \\
\hline$n$ & \multicolumn{6}{|c|}{15} \\
\hline Alt. $\backslash$ Test & $W$ & $\hat{T}_{15 ; c_{2}}^{(-0.7,1)}$ & $\hat{T}_{15}^{(-1.3,2)}$ & $\hat{T}_{15}^{(-0.99,3)}$ & $\hat{T}_{15}^{(0.1,4)}$ & $\hat{T}_{15}^{(0.5,5)}$ \\
\hline 1 & 28.48 & 30.73 & 27.56 & 19.61 & 35.24 & 32.63 \\
\hline 4 & 9.73 & 14.18 & 12.14 & 8.03 & 18.15 & 17.47 \\
\hline 7 & 4.52 & 6.45 & 5.34 & 3.85 & 9.23 & 9.22 \\
\hline 15 & 9.84 & 10.69 & 11.01 & 11.07 & 7.87 & 7.38 \\
\hline 18 & 20.91 & 21.22 & 23.45 & 23.55 & 17.72 & 17.34 \\
\hline 21 & 22.51 & 23.87 & 24.61 & 24.24 & 17.57 & 16.78 \\
\hline 24 & 43.61 & 44.30 & 46.04 & 45.59 & 38.13 & 37.05 \\
\hline 27 & 36.16 & 36.70 & 37.70 & 36.99 & 32.98 & 32.33 \\
\hline 30 & 19.08 & 20.13 & 21.67 & 21.46 & 15.55 & 14.94 \\
\hline 32 & 76.30 & 74.12 & 77.16 & 77.42 & 73.19 & 73.18 \\
\hline Av. & 27.11 & 28.24 & 28.67 & 27.18 & 26.56 & 25.83 \\
\hline$n$ & \multicolumn{6}{|c|}{20} \\
\hline Alt. \Test & $W$ & $\hat{T}_{20 ; c_{4}}^{(-0.01,1)}$ & $\hat{T}_{20}^{(-1.1,2)}$ & $\hat{T}_{20}^{(-0.95,3)}$ & $\hat{T}_{20}^{(0.1,4)}$ & $\hat{T}_{20}^{(0.7,5)}$ \\
\hline 1 & 44.10 & 41.38 & 32.04 & 34.46 & 48.92 & 45.70 \\
\hline 4 & 14.74 & 17.53 & 13.05 & 13.71 & 25.19 & 23.86 \\
\hline 7 & 5.47 & 6.29 & 4.83 & 5.40 & 11.52 & 10.88 \\
\hline 15 & 11.61 & 13.22 & 13.98 & 12.93 & 9.74 & 8.88 \\
\hline 18 & 25.92 & 27.90 & 30.59 & 30.67 & 25.91 & 24.61 \\
\hline 21 & 28.74 & 30.60 & 32.42 & 30.68 & 24.04 & 21.56 \\
\hline 24 & 54.40 & 55.65 & 58.20 & 56.53 & 50.52 & 48.10 \\
\hline 27 & 45.16 & 45.97 & 47.11 & 45.95 & 42.46 & 40.58 \\
\hline 30 & 24.09 & 25.98 & 27.98 & 26.63 & 21.36 & 19.53 \\
\hline 32 & 86.77 & 84.91 & 87.89 & 88.11 & 86.40 & 85.94 \\
\hline Av. & 34.10 & 34.94 & 34.81 & 34.51 & 34.61 & 32.96 \\
\hline$n$ & \multicolumn{6}{|c|}{30} \\
\hline Alt. \Test & $W$ & $\hat{T}_{30 ; c_{1}}^{(-1.3,1)}$ & $\hat{T}_{30 ; c_{1}}^{(-1.3,2)}$ & $\hat{T}_{30}^{(-0.6,3)}$ & $\hat{T}_{30}^{(0.1,4)}$ & $\hat{T}_{30}^{(0.5,5)}$ \\
\hline 1 & 73.01 & 56.34 & 75.97 & 74.69 & 69.91 & 66.34 \\
\hline 4 & 27.37 & 27.60 & 43.10 & 42.26 & 37.57 & 36.15 \\
\hline 7 & 7.85 & 9.15 & 16.62 & 17.07 & 15.27 & 15.36 \\
\hline 15 & 14.80 & 17.31 & 15.02 & 13.15 & 12.66 & 11.69 \\
\hline 18 & 35.69 & 39.01 & 39.91 & 39.38 & 39.91 & 38.37 \\
\hline 21 & 39.73 & 44.24 & 39.99 & 35.59 & 33.94 & 31.46 \\
\hline 24 & 70.20 & 72.93 & 70.55 & 67.96 & 66.36 & 64.31 \\
\hline 27 & 59.21 & 60.60 & 58.61 & 56.34 & 55.40 & 54.47 \\
\hline 30 & 32.56 & 37.12 & 34.45 & 31.64 & 30.62 & 28.75 \\
\hline 32 & 95.81 & 95.65 & 96.39 & 96.52 & 96.63 & 96.56 \\
\hline Av. & 45.62 & 46.00 & 49.06 & 47.46 & 45.83 & 44.35 \\
\hline
\end{tabular}


Table 6 (cont.)

\begin{tabular}{|c|c|c|c|c|c|c|}
\hline$n$ & \multicolumn{5}{|c|}{50} \\
\hline Alt. $\backslash$ Test & $W$ & $\hat{T}_{50 ; c_{4}}^{(-0.99,1)}$ & $\hat{T}_{50}^{(-1.45,2)}$ & $\hat{T}_{50 ; c_{4}}^{(-1,3)}$ & $\hat{T}_{50}^{(-0.1,4)}$ & $\hat{T}_{50}^{(0.5,5)}$ \\
\hline 1 & 97.14 & 83.91 & 87.27 & 91.27 & 91.97 & 90.24 \\
4 & 57.98 & 54.90 & 57.51 & 62.25 & 62.47 & 59.01 \\
7 & 15.11 & 19.32 & 21.57 & 25.54 & 24.98 & 23.52 \\
15 & 19.28 & 24.93 & 23.34 & 19.67 & 18.56 & 16.55 \\
18 & 52.11 & 57.56 & 58.85 & 59.02 & 61.18 & 59.87 \\
21 & 56.08 & 62.03 & 59.32 & 50.14 & 49.77 & 45.34 \\
24 & 86.95 & 89.03 & 88.19 & 83.74 & 84.07 & 81.84 \\
27 & 76.59 & 78.55 & 77.48 & 72.65 & 73.25 & 70.97 \\
30 & 46.92 & 53.31 & 52.34 & 47.17 & 46.78 & 43.49 \\
32 & 99.64 & 99.65 & 99.68 & 99.69 & 99.79 & 99.80 \\
\hline Av. & 60.78 & 62.32 & 62.55 & 61.11 & 61.28 & 59.06 \\
\hline \hline$n$ & \multicolumn{7}{|c|}{70} & & \\
\hline Alt. $\backslash$ Test & $W$ & $\hat{T}_{70 ; c_{4}}^{(-0.99,1)}$ & $\hat{T}_{70 ; c_{1}}^{(-1.3,2)}$ & $\hat{T}_{70}^{(-0.6,3)}$ & $\hat{T}_{70 ; c_{3}}^{(-0.3,4)}$ & $\hat{T}_{70 ; c_{3}}^{(-0.1,5)}$ \\
\hline 1 & 99.89 & 94.91 & 99.55 & 99.30 & 95.94 & 92.87 \\
4 & 82.28 & 76.59 & 89.77 & 85.79 & 89.67 & 86.72 \\
7 & 25.76 & 32.18 & 46.96 & 41.55 & 53.99 & 51.60 \\
15 & 24.30 & 30.88 & 27.97 & 25.32 & 18.78 & 17.29 \\
18 & 65.24 & 70.21 & 75.04 & 76.00 & 63.11 & 61.46 \\
21 & 67.75 & 73.57 & 68.81 & 64.52 & 58.17 & 56.11 \\
24 & 94.11 & 95.56 & 94.35 & 93.29 & 91.48 & 90.97 \\
27 & 86.67 & 87.91 & 86.09 & 84.94 & 82.77 & 82.27 \\
30 & 58.10 & 65.52 & 64.32 & 61.38 & 52.74 & 50.93 \\
32 & 99.98 & 99.96 & 99.99 & 99.99 & 99.97 & 99.97 \\
\hline Av. & 70.41 & 72.73 & 75.28 & 73.21 & 70.66 & 69.02 \\
\hline
\end{tabular}

Table 7. Skew MS populations; sample size $n=10,15,20,30,50,70$; estimates of power of $W$ and $T$ based on 100000 replications

\begin{tabular}{|c|r|r|r|c|c|c|}
\hline$n$ & \multicolumn{6}{|c|}{10} \\
\hline Alt. $\backslash$ Test & \multicolumn{1}{|c|}{$W$} & $\hat{T}_{10 ; c_{4}}^{(1.4,1)}$ & $\hat{T}_{10}^{(0.1,2)}$ & $\hat{T}_{10 ; c_{1}}^{(-1.45,3)}$ & $\hat{T}_{10 ; c_{1}}^{(-1.25,4)}$ & $\hat{T}_{10 ; c_{1}}^{(-0.59,5)}$ \\
\hline 36 & 32.92 & 20.27 & 19.00 & 21.40 & 26.09 & 28.72 \\
38 & 13.13 & 5.65 & 7.56 & 11.76 & 11.08 & 10.21 \\
40 & 13.48 & 16.08 & 15.98 & 14.42 & 14.94 & 15.24 \\
42 & 47.27 & 44.19 & 44.22 & 45.01 & 46.80 & 45.66 \\
44 & 8.31 & 11.34 & 11.30 & 10.10 & 9.41 & 9.33 \\
50 & 23.74 & 28.97 & 28.59 & 26.25 & 26.23 & 25.98 \\
52 & 27.77 & 32.89 & 33.34 & 30.21 & 26.52 & 23.64 \\
54 & 40.06 & 40.84 & 40.93 & 40.83 & 39.03 & 36.52 \\
56 & 89.68 & 82.02 & 80.26 & 83.54 & 86.46 & 87.92 \\
58 & 60.65 & 61.49 & 60.25 & 60.34 & 61.98 & 62.62 \\
\hline Av. & 35.70 & 34.37 & 34.14 & 34.39 & 34.85 & 34.58 \\
\hline \multicolumn{7}{|r|}{}
\end{tabular}


Table 7 (cont.)

\begin{tabular}{|c|c|c|c|c|c|c|}
\hline$n$ & \multicolumn{6}{|c|}{15} \\
\hline Alt. \Test & $W$ & $\hat{T}_{15}^{(-0.5,1)}$ & $\hat{T}_{15}^{(0.1,2)}$ & $\hat{T}_{15 ; c_{1}}^{(-1.45,3)}$ & $\hat{T}_{15 ; c_{1}}^{(-1.25,4)}$ & $\hat{T}_{15 ; c_{1}}^{(-0.99,5)}$ \\
\hline 36 & 53.49 & 42.29 & 24.83 & 29.56 & 38.13 & 42.63 \\
\hline 38 & 20.89 & 11.76 & 18.27 & 19.89 & 17.12 & 15.78 \\
\hline 40 & 21.11 & 20.64 & 21.76 & 21.02 & 22.84 & 24.18 \\
\hline 42 & 71.82 & 70.06 & 61.97 & 66.76 & 69.89 & 69.36 \\
\hline 44 & 11.47 & 11.90 & 14.47 & 13.21 & 12.77 & 13.20 \\
\hline 50 & 38.79 & 39.14 & 41.63 & 40.30 & 41.03 & 41.16 \\
\hline 52 & 42.69 & 44.71 & 44.84 & 43.05 & 36.54 & 32.68 \\
\hline 54 & 54.98 & 55.30 & 54.89 & 54.95 & 51.60 & 48.58 \\
\hline 56 & 98.70 & 97.33 & 94.29 & 95.89 & 97.30 & 97.81 \\
\hline 58 & 82.48 & 81.19 & 79.78 & 80.28 & 82.61 & 83.25 \\
\hline Av. & 49.64 & 47.43 & 45.67 & 46.49 & 46.98 & 46.86 \\
\hline$n$ & \multicolumn{6}{|c|}{20} \\
\hline Alt. $\backslash$ Test & $W$ & $\hat{T}_{20 ; c_{4}}^{(-1.45,1)}$ & $\hat{T}_{20}^{(-0.95,2)}$ & $\hat{T}_{20 ; c_{1}}^{(-1.49,3)}$ & $\hat{T}_{20 ; c_{1}}^{(-1.4,4)}$ & $\hat{T}_{20 ; c_{1}}^{(-0.9,5)}$ \\
\hline 36 & 72.24 & 26.13 & 64.09 & 39.11 & 55.81 & 48.34 \\
\hline 38 & 30.33 & 19.93 & 13.01 & 27.65 & 20.24 & 22.18 \\
\hline 40 & 30.24 & 25.68 & 24.27 & 28.41 & 33.61 & 30.22 \\
\hline 42 & 86.86 & 67.77 & 82.00 & 81.69 & 83.62 & 83.83 \\
\hline 44 & 15.32 & 18.03 & 11.81 & 16.50 & 16.74 & 15.90 \\
\hline 50 & 52.91 & 52.81 & 42.75 & 53.09 & 54.59 & 51.87 \\
\hline 52 & 54.54 & 59.02 & 50.92 & 51.37 & 38.24 & 41.40 \\
\hline 54 & 65.66 & 66.07 & 65.16 & 64.30 & 55.35 & 58.88 \\
\hline 56 & 99.90 & 98.51 & 99.34 & 99.24 & 99.67 & 99.47 \\
\hline 58 & 93.16 & 89.77 & 88.16 & 91.21 & 93.21 & 91.86 \\
\hline Av. & 60.12 & 52.37 & 54.15 & 55.26 & 55.11 & 54.40 \\
\hline$n$ & \multicolumn{6}{|c|}{30} \\
\hline Alt. $\backslash$ Test & $W$ & $\hat{T}_{30}^{(-0.5,1)}$ & $\hat{T}_{30}^{(-0.9,2)}$ & $\hat{T}_{30 ; c_{1}}^{(-1.49,3)}$ & $\hat{T}_{30 ; c_{1}}^{(-1.47,4)}$ & $\hat{T}_{30 ; c_{3}}^{(-0.8)}$ \\
\hline 36 & 92.76 & 81.90 & 86.85 & 51.95 & 77.46 & 68.09 \\
\hline 38 & 51.76 & 19.59 & 21.45 & 42.23 & 26.39 & 44.94 \\
\hline 40 & 49.31 & 48.50 & 43.25 & 41.12 & 51.88 & 40.15 \\
\hline 42 & 97.84 & 97.40 & 96.82 & 94.57 & 95.46 & 92.19 \\
\hline 44 & 23.50 & 25.34 & 19.33 & 22.90 & 24.57 & 19.02 \\
\hline 50 & 74.96 & 74.68 & 65.75 & 70.70 & 74.80 & 70.50 \\
\hline 52 & 71.42 & 74.45 & 67.65 & 63.68 & 43.60 & 62.00 \\
\hline 54 & 79.30 & 79.48 & 79.20 & 77.40 & 61.95 & 78.14 \\
\hline 56 & 100.00 & 100.00 & 99.99 & 99.96 & 100.00 & 100.00 \\
\hline 58 & 99.18 & 98.90 & 98.14 & 98.25 & 99.01 & 98.69 \\
\hline Av. & 74.00 & 70.02 & 67.84 & 66.28 & 65.51 & 67.37 \\
\hline$n$ & \multicolumn{6}{|c|}{50} \\
\hline Alt. \Test & $W$ & $\hat{T}_{50}^{(-1.1,1)}$ & $\hat{T}_{50}^{(-0.95,2)}$ & $\hat{T}_{50}^{(-0.95,3)}$ & $\hat{T}_{50 ; c_{3}}^{(-0.9,4)}$ & $\hat{T}_{50 ; c_{3}}^{(-0.9,5)}$ \\
\hline 36 & 99.83 & 97.46 & 98.91 & 97.33 & 87.65 & 93.14 \\
\hline 38 & 84.00 & 32.01 & 35.53 & 74.25 & 64.57 & 53.18 \\
\hline
\end{tabular}


Table 7 (cont.)

\begin{tabular}{|c|r|r|r|r|r|r|}
\hline$n$ & \multicolumn{7}{|c|}{50} \\
\hline Alt. \Test & \multicolumn{1}{|c|}{$W$} & $\hat{T}_{50}^{(-1.1,1)}$ & $\hat{T}_{50}^{(-0.95,2)}$ & $\hat{T}_{50}^{(-0.95,3)}$ & $\hat{T}_{50 ; c_{3}}^{(-0.4)}$ & $\hat{T}_{50 ; c_{3}}^{(-0.5)}$ \\
\hline 40 & 79.39 & 75.01 & 70.93 & 59.79 & 68.47 & 73.99 \\
42 & 99.97 & 99.95 & 99.94 & 99.46 & 99.67 & 99.76 \\
44 & 41.51 & 40.91 & 34.90 & 27.34 & 37.22 & 39.34 \\
50 & 94.81 & 93.58 & 90.48 & 88.17 & 92.67 & 94.23 \\
52 & 88.01 & 90.23 & 87.98 & 84.41 & 82.88 & 79.98 \\
54 & 92.57 & 92.59 & 92.59 & 92.10 & 92.10 & 91.45 \\
56 & 100.00 & 100.00 & 100.00 & 100.00 & 100.00 & 100.00 \\
58 & 100.00 & 99.98 & 99.97 & 99.96 & 99.98 & 99.99 \\
\hline Av. & 88.01 & 82.17 & 81.12 & 82.28 & 82.52 & 82.51 \\
\hline \hline$n$ & \multicolumn{7}{|c|}{70} & & \\
\hline Alt. \Test & $W$ & $\hat{T}_{70}^{(-0.5,1)}$ & $\hat{T}_{70}^{(-0.9,2)}$ & $\hat{T}_{70}^{(-0.9,3)}$ & $\hat{T}_{70 ; c}^{(-0.9,4)}$ & $\hat{T}_{70 ; 3}^{(-0.8,5)}$ \\
\hline 36 & 100.00 & 99.77 & 99.93 & 99.91 & 96.37 & 97.42 \\
38 & 96.72 & 46.33 & 82.21 & 91.31 & 79.63 & 82.49 \\
40 & 94.01 & 89.89 & 87.51 & 85.07 & 85.56 & 85.98 \\
42 & 100.00 & 100.00 & 100.00 & 99.99 & 99.98 & 100.00 \\
44 & 58.60 & 55.16 & 48.77 & 44.04 & 52.63 & 51.18 \\
50 & 99.28 & 98.68 & 97.64 & 97.68 & 98.39 & 98.51 \\
52 & 94.89 & 96.01 & 94.23 & 92.45 & 91.31 & 90.50 \\
54 & 97.34 & 97.34 & 97.38 & 97.13 & 96.94 & 96.77 \\
56 & 100.00 & 100.00 & 100.00 & 100.00 & 100.00 & 100.00 \\
58 & 100.00 & 100.00 & 100.00 & 100.00 & 100.00 & 100.00 \\
\hline Av. & 94.08 & 88.32 & 90.77 & 90.76 & 90.08 & 90.28 \\
\hline
\end{tabular}

4. Comments on results of Tables 1-7. From Table 1 we conclude that for the large set of $\mathrm{PAB}$ symmetric alternatives our tests are better than the test $W$ and they should be recommended to test normality in the cases $n=10,15,20,30,50,70$.

Table 2 shows that the test $W$ is stronger than our tests under the set of PAB skew alternatives. Averages of powers indicate the quantities of the difference between them for $n=10,15,20,30,50,70$.

Kallenberg and Ledwina $[\mathrm{KL}$ and Cabaña and Cabaña $[\mathrm{CC}$ used ten symmetric alternatives in their comparisons of powers. The results of Table 3 recommend the test $W$ for $n=10$ while for $n=15,20,30,50,70$ they recommend our tests. Kallenberg and Ledwina [KL] used only selected skew alternatives from the set of $\mathrm{PAB}$ skew alternatives in their comparisons. Table 4 shows that in this case the average powers of our tests for $n=$ $10,15,20,30,50,70$ are better than the average powers of the test $W$.

Cabaña and Cabaña [CC] did their comparisons with selected skew alternatives from the set of $\mathrm{PAB}$ skew alternatives. The results of Table 5 
strongly recommend our tests to test normality for $n=10,15,20,30,50,70$ under such selected skew alternatives.

Morris and Szynal MS2 selected ten symmetric alternatives from PAB nonnormal populations. The results of Table 6 recommend our tests for $n=$ $10,15,20,30,50,70$. Table 7 shows that for the choice of skew alternatives by Morris and Szynal MS2 the test $W$ is better than ours.

5. Final conclusions. Here we collect the best tests under different sets of symmetric and skew alternatives.

We write

- $W(200)$ - the test $W$ presented in Pearson et al. $\mathrm{PE}$ for 200 replications

- $W\left(10^{4}\right)$ - the test $W$ presented in Kallenberg and Ledwina KL for 10000 replications

- $W\left(10^{5}\right)$ - the test $W$ presented here for 100000 replications

- $T\left(10^{5}\right)$ - the test presented in Szynal [S] for 100000 replications.

Our investigation gives, among other things, three important statements. In the case of symmetric alternatives the proposed test $T$ is strongly recommended. For skew alternatives the test $T$ is better than $W$ only for some sets of alternatives. It is known that the test $W$ can be applied only under some restrictions. So in the general setting we can recommend our test $T$ with $k=1,2,3$. This conclusion could be confirmed additionally by filling in the empty places in Tables 8 and 9 .

Table 8. Summary performance of tests under sets of symmetric alternatives

\begin{tabular}{|c|c|c|c|c|c|c|c|}
\hline Sym. alt. & \multicolumn{3}{|c|}{ PAB } & \multicolumn{4}{|c|}{$\mathrm{KL}$ and $\mathrm{CC}$} \\
\hline$n /$ Tests & $W(200)$ & $W\left(10^{5}\right)$ & $T\left(10^{5}\right)$ & $W(200)$ & $W\left(10^{4}\right)$ & $W\left(10^{5}\right)$ & $T\left(10^{5}\right)$ \\
\hline & & & $\hat{T}_{10}^{(-1.01,3)}$ & & & & $\hat{T}_{10}^{(-1.01,3)}$ \\
\hline 10 & - & 20.8 & $\begin{array}{c}20.8 \\
\hat{T}_{15}^{(-1.3,2)}\end{array}$ & - & - & 21.2 & $\begin{array}{c}20.2 \\
\hat{T}_{151 . c_{1}}^{(-1.2)}\end{array}$ \\
\hline 15 & - & 29.0 & $\begin{array}{c}30.0 \\
\hat{T}_{20}^{(-1.25,2)}\end{array}$ & - & - & 30.2 & $\begin{array}{c}30.4 \\
\hat{T}_{20 ; c_{1}}^{(-1.25,2)}\end{array}$ \\
\hline 20 & 33.7 & 36.1 & $\begin{array}{c}37.9 \\
\hat{T}_{30 ; c_{1}}^{(-1.2)}\end{array}$ & 38.4 & 39.0 & 38.6 & $\begin{array}{c}39.6 \\
\hat{T}_{30 ; c_{1}}^{(-1.49,1)}\end{array}$ \\
\hline 30 & - & 47.6 & $\begin{array}{c}50.8 \\
\hat{T}_{50 ; c_{1}}^{(-1.3,2)}\end{array}$ & - & - & 52.8 & $\begin{array}{c}53.7 \\
\hat{T}_{50 ; c 1}^{(-1.35,2)}\end{array}$ \\
\hline 50 & 56.8 & 63.0 & $\begin{array}{c}66.9 \\
\hat{T}_{70.3,2)}^{(-1.3)}\end{array}$ & 68.3 & 69.5 & 70.2 & $\begin{array}{c}72.4 \\
\hat{T}_{70.1 .2)}^{(-1.3,2)}\end{array}$ \\
\hline 70 & - & 72.3 & 75.5 & - & - & 79.3 & 80.7 \\
\hline
\end{tabular}


Table 8 (cont.)

\begin{tabular}{|c|c|c|c|}
\hline Sym. alt. & \multicolumn{3}{|c|}{ MS } \\
\hline$n /$ Tests & $W(200)$ & $W\left(10^{5}\right)$ & $T\left(10^{5}\right)$ \\
\hline \multirow{2}{*}{10} & & & $\hat{T}_{10}^{(-1.25,2)}$ \\
& - & 19.4 & 19.6 \\
15 & - & 27.1 & $\hat{T}_{15}^{(-1.3,2)}$ \\
& & & 28.7 \\
& & & $\hat{T}_{20 ; c_{4}}^{(-0.01,1)}$ \\
20 & 34.2 & 34.1 & 34.9 \\
& & & $\hat{T}_{30 ; c_{1}}^{(-1.3,2)}$ \\
30 & - & 45.6 & 49.1 \\
& & & $\hat{T}_{50}^{(-1.45,2)}$ \\
50 & 57.6 & 60.8 & 62.6 \\
& & & $\hat{T}_{70 ; c_{1}}^{(-1.3,2)}$ \\
70 & - & 70.4 & 75.3 \\
\hline
\end{tabular}

Table 9. Summary performance of tests under sets of skew alternatives

\begin{tabular}{|c|c|c|c|c|c|c|c|}
\hline Skew alt. & \multicolumn{3}{|c|}{$\mathrm{PAB}$} & \multicolumn{4}{|c|}{$\mathrm{KL}$} \\
\hline$n /$ Tests & $W(200)$ & $W\left(10^{5}\right)$ & $T\left(10^{5}\right)$ & $W(200)$ & $W\left(10^{4}\right)$ & $W\left(10^{5}\right)$ & $T\left(10^{5}\right)$ \\
\hline 10 & - & 33.4 & $\begin{array}{c}\hat{T}_{10 ; c_{1}}^{(-1.25,4)} \\
32.5 \\
\hat{T}_{15}^{(-0.5,1)}\end{array}$ & - & - & 24.9 & $\begin{array}{c}\hat{T}_{10 ; c_{4}}^{(1.35,1)} \\
26.5 \\
\hat{T}_{15}^{(1.25,1)}\end{array}$ \\
\hline 15 & - & 45.7 & $\begin{array}{c}44.6 \\
\hat{T}_{20}^{(-0.5,1)}\end{array}$ & - & - & 36.7 & $\begin{array}{c}36.9 \\
\hat{T}_{20}^{(-0.5,1)}\end{array}$ \\
\hline 20 & 53.6 & 54.2 & $\begin{array}{c}53.3 \\
\hat{T}_{30}^{(-0.5,1)}\end{array}$ & 45.6 & 46.9 & 46.3 & $\begin{array}{c}46.5 \\
\hat{T}_{30}^{(-0.5,1)}\end{array}$ \\
\hline 30 & - & 65.0 & $\begin{array}{c}63.7 \\
\hat{T}_{50}^{(0.1,2)}\end{array}$ & - & - & 59.7 & $\begin{array}{c}60.3 \\
\hat{T}_{50}^{(-0.5,1)}\end{array}$ \\
\hline 50 & 75.9 & 76.7 & $\begin{array}{c}73.7 \\
\hat{T}_{70}^{(-0.95,2)}\end{array}$ & 72.7 & 73.1 & 75.1 & $\begin{array}{c}75.5 \\
\hat{T}_{70}^{(-0.5,1)}\end{array}$ \\
\hline 70 & - & 83.3 & 80.8 & - & - & 83.8 & 84.0 \\
\hline Skew alt. & \multicolumn{4}{|c|}{$\mathrm{CC}$} & \multicolumn{3}{|c|}{ MS } \\
\hline$n /$ Tests & $W(200)$ & $W\left(10^{4}\right)$ & $W\left(10^{5}\right)$ & $T\left(10^{5}\right)$ & $W(200)$ & $W\left(10^{5}\right)$ & $T\left(10^{5}\right)$ \\
\hline 10 & - & - & 24.3 & $\begin{array}{c}\hat{T}_{10 ; c_{4}}^{(1.4,1)} \\
27.3 \\
\hat{T}_{15}^{(1.4,1)}\end{array}$ & - & 35.7 & $\begin{array}{c}\hat{T}_{10 ; c_{1}}^{(-1.25,4)} \\
34.9 \\
\hat{T}_{15}^{(-0.5,1)}\end{array}$ \\
\hline 15 & - & - & 35.4 & $\begin{array}{c}38.1 \\
\hat{T}_{20}^{(1.35,1)}\end{array}$ & - & 49.6 & $\begin{array}{c}15 \\
47.4 \\
\hat{T}_{20 ; c_{1}}^{(-1.49,3)}\end{array}$ \\
\hline 20 & 43.4 & 44.7 & 44.3 & $\begin{array}{c}46.4 \\
\hat{T}_{30 ; c_{3}}^{(0.1,2)}\end{array}$ & 59.6 & 60.1 & $\begin{array}{c}55.3 \\
\hat{T}_{30}^{(-0.5,1)}\end{array}$ \\
\hline 30 & - & - & 57.2 & $\begin{array}{c}59.3 \\
\hat{T}_{50.2,2)}^{(0.2)}\end{array}$ & - & 74.0 & $\begin{array}{c}70.0 \\
\hat{T}_{50.9,4)}^{(-0.9}\end{array}$ \\
\hline 50 & 70.6 & 73.9 & 73.2 & $\begin{array}{c}74.4 \\
\hat{T}_{70}^{(-0.5,1)}\end{array}$ & 88.3 & 88.0 & $\begin{array}{c}82.5 \\
\hat{T}_{70}^{(-0.9,2)}\end{array}$ \\
\hline 70 & - & - & 82.5 & 82.8 & - & 94.1 & 90.8 \\
\hline
\end{tabular}




\section{References}

[CC] A. Cabaña and E. M. Cabaña, Tests of normality based on transformed empirical processes, Methodology Comput. Appl. Probab. 5 (2003), 309-335.

[KL] W. C. M. Kallenberg and T. Ledwina, Data driven smooth tests for composite hypotheses: comparison of powers, J. Statist. Comput. Simul. 59 (1997), 101-121.

[MS1] M. Morris and D. Szynal, Goodness-of-fit tests based on characterizations involving moments of order statistics, J. Pure Appl. Math. 38 (2007), 83-121.

[MS2] M. Morris and D. Szynal, Some U-statistics in goodness-of-fit tests derived from characterizations via record values, J. Pure Appl. Math. 4 (2008), 507-582.

[S] D. Szynal, Goodness-of-fit tests derived from characterizations of continuous distributions, in: Banach Center Publ. 90, Inst. Math., Polish Acad. Sci., 2010, 203223.

[SW1] D. Szynal and W. Wołyński, Simulation supplement to goodness-of-fit tests derived from characterizations of continuous distributions via record values, J. Pure Appl. Math. 87 (2013), 629-644.

[SW2] D. Szynal and W. Wołyński, On two families of tests for normality with empirical description of their performances, Discussiones Math. Probab. Statist. 34 (2014), 169-185.

[PE] E. S. Pearson, R. B. D'Agostino and K. O. Bowman, Tests for departure from normality: Comparison of powers, Biometrika 64 (1977), 231-246.

Dominik Szynal

Institute of Mathematics

Maria Curie-Skłodowska University

20-031 Lublin, Poland

E-mail: szynal@poczta.umcs.lublin.pl
Waldemar Wołyński Faculty of Mathematics and Computer Science Adam Mickiewicz University 61-614 Poznań, Poland E-mail: wolynski@amu.edu.pl 
